PDES, SUBMANIFOLDS AND

AFFINE DIFFERENTIAL GEOMETRY

BANACH CENTER PUBLICATIONS, VOLUME 69

INSTITUTE OF MATHEMATICS

POLISH ACADEMY OF SCIENCES

WARSZAWA 2005

\title{
ALGEBRAIC REPRESENTATION FORMULAS FOR NULL CURVES IN $\mathrm{Sl}(2, \mathbb{C})$
}

\author{
HUBERT GOLLEK \\ Institute of Mathematics, Humboldt University \\ 10099 Berlin, Germany \\ Sitz: Rudower Chaussee 25 \\ E-mail:gollek@mathematik.hu-berlin.de
}

\begin{abstract}
We study curves in $\mathrm{Sl}(2, \mathbb{C})$ whose tangent vectors have vanishing length with respect to the biinvariant conformal metric induced by the Killing form, so-called null curves. We establish differential invariants of them that resemble infinitesimal arc length, curvature and torsion of ordinary curves in Euclidean 3-space. We discuss various differential-algebraic representation formulas for null curves. One of them, a modification of the Bianchi-Small formula, gives an $\mathrm{Sl}(2, \mathbb{C})$-equivariant bijection between pairs of meromorphic functions and null curves. The inverse of this formula is also differential-algebraic. The other one is based on an integral formula deduced from that of R. Bryant, using certain natural differential operators on Riemannian surfaces that we introduced in [7] for differential-algebraic representation formulas of curves in $\mathbb{C}^{3}$. We demonstrate some commands of a Mathematica package that resulted from our investigations, containing algebraic and graphical utilities to handle null curves, their invariants, representation formulas and associated surfaces of constant mean curvature 1 in $\mathbb{H}^{3}$, taking into consideration several models of $\mathbb{H}^{3}$.
\end{abstract}

1. Introduction. Let $\Sigma$ be a Riemannian surface and $\mathbf{N}_{\Sigma}$ the set of all null curves of $\operatorname{Sl}(2, \mathbb{C})$ defined on $\Sigma$, i.e., the set of all meromorphic maps $\mathrm{F}: \Sigma \rightarrow \operatorname{Sl}(2, \mathbb{C})$ such that $\langle\mathrm{dF}, \mathrm{dF}\rangle=0$ for the biinvariant conformal metric $\langle.,$.$\rangle of \mathrm{Sl}(2, \mathbb{C})$. Null curves and their differential invariants are in close relation to surfaces of constant mean curvature 1 (cmc-1-surfaces) in the 3-dimensional hyperbolic space $\mathbb{H}^{3}$. Differential invariants of null curves correspond to differential invariants of cmc-1-surfaces. However, instead of surfaces, we will focus on curves and their invariants.

Roughly speaking, a representation formula for $\mathbf{N}_{\Sigma}$ is an operator $\mathbf{R}$ acting from a certain space $\mathcal{E}$ of sections of a bundle over $\Sigma$ to $\mathbf{N}_{\Sigma}$. In most cases $\mathcal{E}$ is the space $\mathcal{A}_{\Sigma}$ of non-constant meromorphic functions, the space $\mathcal{X}_{\Sigma}$ of meromorphic vector fields, the

2000 Mathematics Subject Classification: Primary 53A10; Secondary 53C42, 68U05.

The paper is in final form and no version of it will be published elsewhere. 
space $\mathcal{L}_{\Sigma}$ of meromorphic 1 -forms or one of the cartesian products $\mathcal{A}_{\Sigma} \times \mathcal{A}_{\Sigma}, \mathcal{A}_{\Sigma} \times \mathcal{X}_{\Sigma}$ or $\mathcal{A}_{\Sigma} \times \mathcal{L}_{\Sigma}$. Often, instead of $\mathbf{N}_{\Sigma}$ other spaces derived from it have to be considered, mainly, the factor space $\mathbf{N}_{\Sigma} / \mathrm{Sl}(2, \mathbb{C})$ by the left or right action of $\operatorname{Sl}(2, \mathbb{C}), \mathbf{N}_{\mathbb{C}}$ modulo reparametrizations, or the subspace $\mathbf{N}_{\mathbb{C}}^{*} \subset \mathbf{N}_{\mathbb{C}}$ of curves in natural parametrization.

We distinguish between integral and algebraic representation formulas. The latter are differential operators that occur in our examples always as rational expressions in their argument functions and derivatives of these. The representation formulas containing integrations are more difficult to handle, but may have simpler expressions and other advantages. Simpler in structure but more difficult to handle than integral formulas is R. Bryant's representation formula

$$
\left\{\begin{array}{l}
\mathbf{B}:(g, \omega) \in \mathcal{A}_{\Sigma} \times \mathcal{L}_{\Sigma} \rightarrow \mathbf{B}_{\mathbf{g}, \omega} \in \mathbf{N}_{\Sigma} / \mathrm{Sl}(2, \mathbb{C}), \text { where } \mathbf{B}_{\mathbf{g}, \omega} \\
\text { is a solution of the linear system } \mathrm{d} \mathbf{B}_{\mathbf{g}, \omega}=\mathbf{B}_{\mathbf{g}, \omega}\left(\begin{array}{cc}
\mathbf{g} & -\mathbf{g}^{2} \\
1 & -\mathbf{g}
\end{array}\right) \omega
\end{array}\right.
$$

involving the solution of a system of ordinary differential equations. This operator is not correctly defined on $\mathbf{N}_{\Sigma}$, even if it is considered as an operator acting from $\mathcal{A}_{\Sigma} \times \mathcal{L}_{\Sigma}$ to $\mathbf{N}_{\Sigma} / \operatorname{Sl}(2, \mathbb{C})$ (left hand side action). In general, solutions of (1.1) as well as of integral representation formulas we are going to consider live on covering spaces of $\Sigma$. This formula has many analogies to the classical Weierstraß formula

$$
(\mathbf{g}, \omega) \in \mathcal{A}_{\Sigma} \times \mathcal{L}_{\Sigma} \rightarrow \mathbf{W}_{\mathbf{g}, \omega}(z)=\int\left(1-\mathbf{g}^{2}(z), i\left(1+\mathbf{g}^{2}(z)\right), 2 \mathbf{g}(z)\right)^{\top} \omega
$$

for null curves in $\mathbb{C}^{3}$. Both, (1.1) and (1.2) are almost bijective and establish a correspondence between null curves in $\mathbb{C}^{3}$ and null curves inSl(2, $\left.\mathbb{C}\right)$. Curves $\mathbf{W}_{\mathbf{g}, \omega}$ and $\mathbf{B}_{\mathbf{g}, \omega}$ that correspond to the same pair $(\mathbf{g}, \omega)$ are called cousins of each other. Some explicit cousin constructions will be shown in section 7.2.

Besides (1.1), representation formulas we are going to consider in this paper are:

- An integral formula Mod : $\mathcal{A}_{\Sigma} \times \mathcal{A}_{\Sigma} \rightarrow \mathbf{N}_{\Sigma}$ obtained by a modification of R. Bryant's formula (see section 4 , equation (4.8)).

- An integral formula Iwa : $\mathcal{A}_{\mathbb{C}} \times \mathcal{A}_{\mathbb{C}} \rightarrow \mathbf{N}_{\mathbb{C}}$ obtained from a meromorphic parametrization $\mathbf{x}: \mathbb{C}^{3} \rightarrow \operatorname{Sl}(2, \mathbb{C})$ (see section 4 , equation $(4.3)$ ) of $\mathrm{Sl}(2, \mathbb{C})$ coming from the Iwasawa decomposition. Under $\mathbf{x}$, (1.1) turns into a simple differential equation for the 3 components of a curve in $\mathbb{C}^{3}$ whose solution gives Iwa.

- A family $\mathbf{M}^{(n, k, l)}: \mathcal{A}_{\Sigma} \times \mathcal{X}_{\Sigma} \rightarrow \mathbf{N}_{\Sigma}\left(n>2, n-1>k \geq 0, l \in\left\{0,1, \ldots,{ }^{\prime} \operatorname{Min}\{k, n-\right.\right.$ $k-1\}\}$ ) of algebraic formulas is obtained by removing integrations occurring in Mod. $\mathbf{M}^{(n, k, l)}$ is almost bijective. It can be inverted by integrations (see section 5 , equation (5.5))

- Finally, a modification of A. Small's formula (see [11], [10] or [13]) leads to an algebraic formula $\mathbf{B S}:(\mathbf{f}, \mathbf{g}) \in \mathcal{A}_{\Sigma} \times \mathcal{A}_{\Sigma} \rightarrow \mathbf{N}_{\Sigma}$ depending on two arguments. For this purpose a partial operation between null curves is defined, assigning to any pair $\left(\mathrm{F}_{1}, \mathrm{~F}_{2}\right)$ of null curves such that their Bryant data $\mathbf{g}_{1}$ and $\mathbf{g}_{2}$ (i.e., the first arguments of (1.1)) are equal, a new null curve $\mathbf{B S}_{\mathbf{f}, \mathbf{g}}=\mathrm{F}_{1}\left(\mathrm{~F}_{2}\right)^{-1} . \mathbf{B S}_{\mathbf{f}, \mathbf{g}}$ is 
invariant with respect to the fractional linear action of $\operatorname{Sl}(2, \mathbb{C})$ on functions of $\mathcal{A}_{\Sigma}$. It coincides with a formula derived in ([9]) by different methods.

On $\mathbf{N}_{\Sigma}$ two differential invariants $\pi^{\prime 2}: \mathbf{N}_{\Sigma} \rightarrow \mathcal{L}_{\Sigma} \otimes \mathcal{L}_{\Sigma}$ and $\kappa^{2}: \mathbf{N}_{\Sigma} \rightarrow \mathcal{A}_{\Sigma}$ are defined with properties similar to those of null curves in $\mathbb{C}^{3}$ (see [5] and [6]). We call $\pi^{\prime}$ the infinitesimal natural parameter and $\kappa$ the null curvature. Together with some kind of torsion, defined as $\tau=i \kappa^{\prime} / \kappa$, these invariants resemble the classical invariants of curves in Euclidean 3 -space. We associate in section 3 with any null curve an orthonormal moving frame and prove an analogue of Frenet's system of differential equations, showing that $\pi$ and $\kappa$ form a complete set of invariants of null curves with respect to the right and left action of $\operatorname{Sl}(2, \mathbb{C})$.

We compute these invariants for the representation formulas listed above, i.e., if $\mathcal{E}$ is the space of functions $\mathcal{E}$ corresponding to one of these formulas, we derive formulas for the compositions $\pi^{\prime 2} \circ \mathbf{R}: \mathcal{E} \rightarrow \mathcal{L}_{\Sigma} \otimes \mathcal{L}_{\Sigma}$ and $\kappa \circ \mathbf{R}: \mathcal{E} \rightarrow \mathcal{A}_{\Sigma}$.

In the appendix we show examples illustrating some commands of a Mathematicapackage $\mathrm{cmc} 1 . \mathrm{m}$ that arose from the necessity to collect the great amount of formulas and operations concerning null curves in electronic code. This package is still unfinished. It will be completed and improved. It contains some more material than is discussed here, for instance integral representation formulas for curves with preassigned natural parameter, but also much of technical commands related to cmc-1-surfaces and plotting utilities that produce pictures of the surfaces (in the ball model as well as in the upper half space model of $\mathbb{H}^{3}$ ) with least time needed, depending on the complexity of the parametrization of the corresponding null curve. Still, a manageable formula producing curves with preassigned curvature is missing.

2. The biinvariant conformal metric of $\operatorname{Sl}(2, \mathbb{C})$. The group $\mathrm{Sl}(2, \mathbb{C})$ carries a natural biinvariant conformal metric, i.e., a field $\langle.,$.$\rangle of symmetric, nondegenerate \mathbb{C}$-bilinear forms on the tangent bundle, defined by left-translation of $1 / 8$ of the Killing form $B$ of the Lie algebra $\operatorname{sl}(2, \mathbb{C})$ to the points of $\operatorname{Sl}(2, \mathbb{C})$. Explicitly, for $X, Y \in \operatorname{sl}(2, \mathbb{C})$ and $g \in \operatorname{Sl}(2, \mathbb{C}):$

$$
\left\{\begin{array}{l}
B(X, Y)=\operatorname{tr}(\operatorname{ad} X \circ \operatorname{ad} Y)=8 x_{11} y_{11}+4 x_{12} y_{21}+4 x_{21} y_{12}, \\
\left\langle l_{g}^{*} X, l_{g}^{*} Y\right\rangle=\frac{B(X, Y)}{8}, \text { where } X=\left(\begin{array}{rr}
x_{11} & x_{12} \\
x_{21} & -x_{11}
\end{array}\right), Y=\left(\begin{array}{rr}
y_{11} & y_{12} \\
y_{21} & -y_{11}
\end{array}\right),
\end{array}\right.
$$

i.e., $B(X, X)=-8 \operatorname{Det}(X)$, and $\langle\mathfrak{t}, \mathfrak{s}\rangle=-B\left(\left(l_{g}^{-1}\right)^{*} \mathfrak{t},\left(l_{g}^{-1}\right)^{*} \mathfrak{s}\right) / 8$ for any two tangent vectors $\mathfrak{t}, \mathfrak{s} \in T_{g}(\operatorname{sl}(2, \mathbb{C}))$

The complex metric $\langle.,$.$\rangle induces a complex connection \nabla$ on the tangent bundle of $\mathrm{Sl}(2, \mathbb{C})$ by the usual formula defining the Levi-Civita connection in the Riemannian case, namely, for arbitrary meromorphic vector fields $X, Y, Z$ on $\mathrm{Sl}(2, \mathbb{C})$ the scalar product $2\left\langle X, \nabla_{Z} Y\right\rangle$ is given by the Koszul formula

$$
Z\langle X, Y\rangle+Y\langle X, Z\rangle-X\langle Y, Z\rangle+\langle Z,[X, Y]\rangle-\langle X,[Y, Z]\rangle+\langle Y,[X, Z]\rangle .
$$

We use the Iwasawa decomposition of $\mathrm{Sl}(2, \mathbb{C})$ to define local coordinates. The following rational map is a parametrization of an open dense part $U \subset \operatorname{Sl}(2, \mathbb{C})$. 


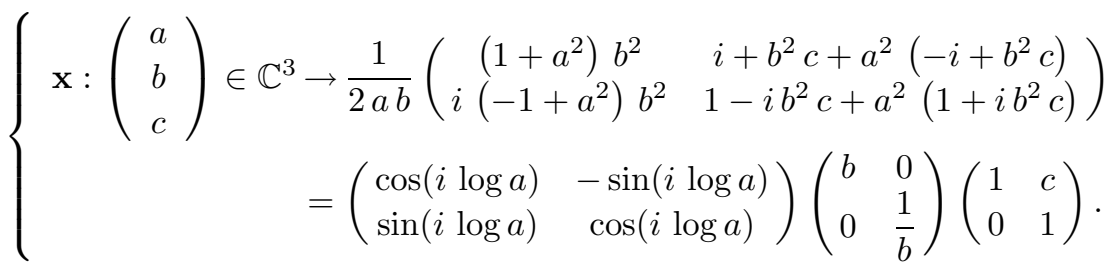

Local inverses of $\mathbf{x}$ can be expressed in terms of square roots. One has

$$
\mathbf{x}^{-1}(\mathrm{~F})=\left(\begin{array}{c} 
\pm \sqrt{A-i C} / \sqrt{A+i C} \\
\pm \sqrt{A-i C} \sqrt{A+i C} \\
\frac{i+A B+i B C-i A D+C D}{A^{2}+C^{2}}
\end{array}\right) \text { for } \mathrm{F}=\left(\begin{array}{ll}
A & B \\
C & D
\end{array}\right) \in \mathrm{Gl}(2, \mathbb{C})
$$

i.e., $\mathbf{x}^{-1}$ is defined as a meromorphic map on $\mathrm{Gl}(2, \mathbb{C})$. If $\mathrm{F} \in \mathrm{Sl}(2, \mathbb{C})$ then the third component of $\mathbf{x}^{-1}(\mathrm{~F})$ becomes $(A B+C D) /\left(A^{2}+B^{2}\right)$.

In terms of these coordinates the metric tensor of $\langle.,$.$\rangle is given by$

$$
\left(g_{i j}\right)_{i, j=1}^{3}=\frac{1}{2 a^{2} b^{2}}\left(\begin{array}{ccc}
2 b^{2} & 0 & i a b^{4} \\
0 & 2 a^{2} & 0 \\
i a b^{4} & 0 & 0
\end{array}\right),
$$

while the matrices $\left(\Gamma_{i j}^{1}\right),\left(\Gamma_{i j}^{2}\right),\left(\Gamma_{i j}^{3}\right)$ of the Christoffel symbols are

$$
\left(\begin{array}{ccc}
\frac{-1}{a} & \frac{1}{b} & 0 \\
\frac{1}{b} & 0 & 0 \\
0 & 0 & 0
\end{array}\right), \quad\left(\begin{array}{ccc}
0 & 0 & \frac{-i b^{3}}{2 a} \\
0 & \frac{-1}{b} & 0 \\
\frac{-i b^{3}}{2 a} & 0 & 0
\end{array}\right), \quad\left(\begin{array}{ccc}
0 & \frac{2 i}{a b^{3}} & 0 \\
2 i & 0 & \frac{1}{b} \\
0 b^{3} & \frac{1}{b} & 0
\end{array}\right)
$$

respectively.

3. Null curves in $\operatorname{Sl}(2, \mathbb{C})$. Let $\Sigma$ be a Riemannian surface. A null curve $\gamma: \Sigma \rightarrow$ $\mathrm{Sl}(2, \mathbb{C})$ is a nonconstant meromorphic map of $\Sigma$ into $\mathbb{C}^{3}$ whose tangent vector has length 0 with respect to the conformal metric $\langle.,$.$\rangle . For a holomorphic curve \gamma(z)$ in $\operatorname{Sl}(2, \mathbb{C})$ define the sequence of higher order tangent vectors by

$$
\mathbf{T}_{1}=\gamma^{\prime}, \quad \mathbf{T}_{n+1}=\nabla_{\mathbf{T}_{1}} \mathbf{T}_{n}
$$

If $\gamma$ is a null curve, then $\left\langle\nabla \mathbf{T}_{1}, \nabla \mathbf{T}_{1}\right\rangle$ can be regarded as a biquadratic differential on $\Sigma$. We define the infinitesimal natural parameter $\pi$ of $\gamma$ by $\pi^{\prime}(z)=\sqrt[4]{\left\langle\nabla \mathbf{T}_{1}, \nabla \mathbf{T}_{1}\right\rangle}$. If $\gamma$ is a full curve, i.e., if $\mathbf{T}_{1}, \mathbf{T}_{2}, \mathbf{T}_{3}$ are linearly independent, then $\pi(z)$ is locally well defined by choosing a definite branch of the square root function and integrating.

DeFinition 3.1. The natural parameter $\pi$ and the null curvature $\kappa$ of a null curve $\gamma$ in $\mathrm{Sl}(2, \mathbb{C})$ are given by

$$
\pi=\int \sqrt[4]{\left\langle\mathbf{T}_{2}, \mathbf{T}_{2}\right\rangle}, \quad \kappa(\pi)=\sqrt{\left\langle\mathbf{T}_{3}(\pi), \mathbf{T}_{3}(\pi)\right\rangle},
$$

where in the second case it is assumed that $\gamma$ is parametrized by its natural parameter. 
Similar to arc length, curvature and torsion in the case of curves in Euclidean space $\mathbb{R}^{3}$, the functions $\pi$ and $\kappa$ form a complete system of invariants, i.e., a null curve is uniquely determined by them up to transformation with elements of $\mathrm{Sl}(2, \mathbb{C})$ from the right and left.

Proposition 3.1. Let $\gamma$ be a full null curve parametrized by its natural parameter $p$, $\mathbf{T}_{1}, \mathbf{T}_{2}, \ldots$ the infinite sequence of it's higher order tangent vectors, and $M_{n}=\left(\mu_{i j}\right)_{i, j=1}^{n}$ a finite part of the matrix of the scalar products $\mu_{i j}(\pi)=\left\langle\mathbf{T}_{i}(\pi), \mathbf{T}_{j}(\pi)\right\rangle$. Then $M_{5}$ is the following matrix:

$$
\left(\begin{array}{ccccc}
0 & 0 & -1 & 0 & \kappa^{2} \\
0 & 1 & 0 & -\kappa^{2} & -3 \kappa \kappa^{\prime} \\
-1 & 0 & \kappa^{2} & \kappa \kappa^{\prime} & \kappa^{2}+\kappa \kappa^{\prime \prime}-\kappa^{4} \\
0 & -\kappa^{2} & \kappa \kappa^{\prime} & \kappa^{4} & 2 \kappa^{3} \kappa^{\prime} \\
\kappa^{2} & -3 \kappa \kappa^{\prime} & \kappa^{\prime 2}+\kappa \kappa^{\prime \prime}-\kappa^{4} & 2 \kappa^{3} \kappa^{\prime} & \kappa^{2}\left(\kappa^{4}+7 \kappa^{\prime 2}-2 \kappa \kappa^{\prime \prime}\right)
\end{array}\right)
$$

Moreover, the 4-th order tangent vector is expressed in terms of the vector fields $\mathbf{T}_{1}, \mathbf{T}_{2}, \mathbf{T}_{3}$ as follows:

$$
\mathbf{T}_{4}(\pi)=-\kappa(\pi) \kappa^{\prime}(\pi) \mathbf{T}_{1}(\pi)-\kappa^{2}(\pi) \mathbf{T}_{2}(\pi)
$$

Proof. Successive differentiation of the equations $\left\langle\mathbf{T}_{1}, \mathbf{T}_{1}\right\rangle=0,\left\langle\mathbf{T}_{2}, \mathbf{T}_{2}\right\rangle=1$ and $\left\langle\mathbf{T}_{3}, \mathbf{T}_{3}\right\rangle$ $=\kappa^{2}$ yields the entries $\mu_{i j}$ for $1 \leq i, j \leq 4$, except $\mu_{44}$. Assuming that $\mathbf{T}_{1}, \mathbf{T}_{2}, \mathbf{T}_{3}$ are linearly independent, scalar multiplication of an ansatz $\mathbf{T}_{4}=a \mathbf{T}_{1}+b \mathbf{T}_{2}+c \mathbf{T}_{3}$ with $\mathbf{T}_{1}, \mathbf{T}_{2}, \mathbf{T}_{3}$ respectively gives 3 equations from which $a, b, c$ can be computed, to obtain (3.3). Next, from (3.3) and the entries computed until now we obtain the entry $\mu_{44}=\left\langle\mathbf{T}_{4}, \mathbf{T}_{4}\right\rangle=\kappa^{4}$. The other entries are obtained by continuing in this way.

Next, we will discuss an analogue of the system of Frenet equations. Let $\gamma$ be a null curve in natural parametrization, defined on $\mathbb{C}$, i.e., we assume that $\left\langle\gamma^{\prime \prime}(z), \gamma^{\prime \prime}(z)\right\rangle=1$. Choosing locally a branch of the square root we define $\kappa(z)=\sqrt{\left\langle\gamma^{\prime \prime \prime}(z), \gamma^{\prime \prime \prime}(z)\right\rangle}$ and an orthonormal moving frame $(\mathbf{T}, \mathbf{N}, \mathbf{B})$ along $\gamma$ by

$$
\mathbf{T}=\mathbf{T}_{2}, \quad \mathbf{N}=\frac{1}{\kappa} \mathbf{T}_{3}, \quad \text { and } \quad \mathbf{B}=i\left(\kappa \mathbf{T}_{1}+\frac{1}{\kappa} \mathbf{T}_{3}\right) .
$$

Proposition 3.2. With these notations, the following analogue of Frenet's equations of $\mathbb{R}^{3}$ holds:

$$
\nabla \mathbf{T}=\kappa \mathbf{N}, \quad \nabla \mathbf{N}=-\kappa \mathbf{T}+\frac{i \kappa^{\prime}}{\kappa} \mathbf{B}, \quad \nabla \mathbf{B}=-\frac{i \kappa^{\prime}}{\kappa} \mathbf{N} .
$$

The proof is straightforward, using the matrix (3.2) of scalar products and (3.3).

Choose a local chart on $\operatorname{Sl}(2, \mathbb{C})$, assume that $z=\pi$ is the natural parameter and combine the tangent vectors $\mathbf{T}(z), \mathbf{N}(z), \mathbf{B}(z)$ to an orthogonal matrix $M(z)$. Then $(3.5)$ becomes a linear system of ordinary differential equations whose solution gives $\mathbf{T}(z)$ and by another integration $F$. In a similar way one could proceed with the vectors $\mathbf{T}_{1}, \mathbf{T}_{2}, \mathbf{T}_{3}$, using equation (3.3) to obtain $\mathbf{T}_{1}$ and in turn F. Therefore, the following existence and uniqueness theorem holds. 
TheORem 3.1. A null curve $\mathrm{F}$ in $\mathrm{Sl}(2, \mathbb{C})$ is completely determined by its natural parameter $\pi$, its curvature $\kappa$ and an initial value $\mathrm{F}\left(z_{0}\right)$. Any two null curves $\mathrm{F}_{1}, \mathrm{~F}_{2}$ with the same invariants are congruent under the two-side action of $\mathrm{Sl}(2, \mathbb{C})$ on itself.

Given a null curve $\mathrm{F}=\left(\begin{array}{ll}A & B \\ C & D\end{array}\right): \Sigma \rightarrow \mathrm{Sl}(2, \mathbb{C})$, consider its Maurer-Cartan derivative $\mathcal{N}=\mathrm{F}^{-1} \mathrm{dF}$. Since the determinant and the trace of $\mathcal{N}$ are zero, there exist a function $g$ and a 1-form $\omega$ such that

$$
\mathcal{N}=\left(\begin{array}{cc}
g & -g^{2} \\
1 & -g
\end{array}\right) \omega
$$

we infer from $\mathcal{N}=\mathrm{F}^{-1} \mathrm{dF}$ that

$$
\left\{\begin{aligned}
\omega & =\mathcal{N}_{21}=A \mathrm{~d} C-C \mathrm{~d} A \text { and } \\
g(z) & =\frac{\mathcal{N}_{11}}{\mathcal{N}_{21}}=\frac{D \mathrm{~d} A-B \mathrm{~d} C}{A \mathrm{~d} C-C \mathrm{~d} A}=\frac{\mathcal{N}_{12}}{\mathcal{N}_{22}}=\frac{D \mathrm{~d} B-B \mathrm{~d} D}{A \mathrm{~d} D-C \mathrm{~d} B} .
\end{aligned}\right.
$$

Conversely, given a meromorphic function $g$ and a meromorphic differential $\omega$ on $\Sigma$, we obtain a null curve $\mathrm{F}$ by solving the system $\mathcal{N}=\mathrm{F}^{-1} \mathrm{dF}$. In general, $\mathrm{F}$ will be defined only locally, or on a covering space of $\Sigma$. We call the pair $(g, \omega)$ the Bryant data of F.

The relation between classes $[\mathrm{F}]=\{L \mathrm{~F} \mid L \in \mathrm{Sl}(2, \mathbb{C})\}$ of left translates of null curves and their Bryant data $(g, \omega)$ is $\operatorname{Sl}(2, \mathbb{C})$-equivariant, if the action of $\operatorname{Sl}(2, \mathbb{C})$ given by $(R,[\mathrm{~F}]) \rightarrow\left[\mathrm{F} R^{-1}\right], R \in \mathrm{Sl}(2, \mathbb{C})$, is considered. Namely, if $R=\left(\begin{array}{ll}a & b \\ c & d\end{array}\right)$, the Bryant data of $\mathrm{F} R^{-1}$ are $(\widetilde{g}, \widetilde{\omega})$ given by

$$
(g, \omega) \rightarrow(\widetilde{g}, \widetilde{\omega})=\left(\frac{a g+b}{c g+d},(c g+d)^{2} \omega\right)
$$

and the assignment $(R,(g, \omega)) \rightarrow(\widetilde{g}, \widetilde{\omega})$ of $(3.8)$ is a left group action of $\operatorname{Sl}(2, \mathbb{C})$ on the space of all such pairs $(g, \omega)$.

Proposition 3.3. The natural parameter $\pi$ and the curvature $\kappa$ of a null curve $\gamma(z)=$ $\mathbf{B}_{g, \omega}(z)$ given by Bryant's formula (1.1) in terms of a meromorphic function $g$ and a meromorphic 1-form $\omega$ are

$$
\left\{\begin{aligned}
\pi^{\prime 4}(z) & =\omega(z)^{2} g^{\prime}(z)^{2} \text { and } \\
\kappa(z) & =-2 \omega(z)^{3} g^{\prime}(z)^{3}+4 g^{\prime}(z)^{2} \omega^{\prime}(z)^{2}+\omega(z)^{2} g^{\prime \prime}(z)^{2}- \\
& =2 \omega(z) g^{\prime}(z)\left(-2 \omega^{\prime}(z) g^{\prime \prime}(z)+g^{\prime}(z) \omega^{\prime \prime}(z)\right)
\end{aligned}\right.
$$

If the parametrization of $\gamma(z)$ is the natural one, i.e., if $\omega(z) g^{\prime}(z)=1$, the null curvature is given by

$$
\kappa(z)=-2-\frac{3 g^{\prime \prime}(z)^{2}}{g^{\prime}(z)^{2}}+\frac{2 g^{(3)}(z)}{g^{\prime}(z)}=-2-(\mathcal{S}(g)(z))^{2},
$$

where $\mathcal{S}(g)(z)=\sqrt{\frac{3 g^{\prime \prime 2}-2 g^{\prime} g^{(3)}}{g^{\prime 2}}}$ is the Schwarzian derivative of $g$.

4. Representation formulas containing integrations only. There exist several possibilities to change the input data $g, \omega$ of the Bryant formula so that the corresponding null curve is given by integrals of the new data. We discuss two of them here. 
First, we consider the parametrization $\mathbf{x}$ defined in $(2.3)$ and the pull back $h(.,)=$. $\mathbf{x}_{*}\langle.,$.$\rangle of the invariant metric of \mathrm{Sl}(2, \mathbb{C})$ to $\mathbb{C}^{3}$. Considering an arbitrary curve $\gamma(z)=(a(z), b(z), c(z))^{\top}$ in $\mathbb{C}^{3}$, the infinitesimal arc length of $\gamma$ can be computed as $h\left(\gamma^{\prime}(z), \gamma^{\prime}(z)\right)=-\frac{1}{8} \operatorname{Det}(\mathcal{N}(z))$, where $\mathcal{N}(z)=\mathrm{F}^{-1}(z) \mathrm{F}^{\prime}(z): \mathbb{C} \rightarrow \operatorname{sl}(2, \mathbb{C})$ is the MaurerCartan derivative of the curve $\mathrm{F}(z)=\mathbf{x}(\gamma(z))$. The resulting expression is

$$
\langle\mathcal{N}(z), \mathcal{N}(z)\rangle=h\left(\gamma^{\prime}(z), \gamma^{\prime}(z)\right)=-\left(\frac{a^{\prime 2}(z)}{a^{2}(z)}+\frac{b^{\prime 2}(z)}{b^{2}(z)}+\frac{i b^{2}(z) a^{\prime}(z) c^{\prime}(z)}{a(z)}\right)
$$

We solve the equation $h\left(\gamma^{\prime}(z), \gamma^{\prime}(z)\right)=0$ for $c^{\prime}(z)$. Integration yields

$$
c(z)=\int \frac{i a(z)}{b^{2}(z) a^{\prime}(z)}\left(\frac{a^{\prime 2}(z)}{a^{2}(z)}+\frac{b^{\prime 2}(z)}{b^{2}(z)}\right) \mathrm{d} z .
$$

In this way we obtain an integral representation formula $(a, b) \rightarrow \operatorname{Iwa}_{a, b}(z)=$ $\mathbf{x}(a(z), b(z), c(z))$, where $a, b$ are arbitrary meromorphic functions and $c$ is given by (4.2). Its explicit form is

$$
\begin{cases}\operatorname{Iwa}_{a, b}(z)=\frac{1}{2 a b}\left(\begin{array}{cc}
\left(a^{2}+1\right) b^{2} & -i\left(a^{2}-1\right)+\left(a^{2}+1\right) b^{2} \int \mu(z) \mathrm{d} z \\
i\left(a^{2}-1\right) b^{2} & a^{2}+1+i\left(a^{2}-1\right) b^{2} \int \mu(z) \mathrm{d} z
\end{array}\right) \\
\text { where } \mu \text { is the differential form } \mu=\frac{i \mathrm{~d} a}{a b^{2}}+\frac{i a \mathrm{~d} b^{2}}{b^{4} \mathrm{~d} a}\end{cases}
$$

The Bryant data of this curve are

$$
g=\frac{-i a(z) b^{\prime}(z)}{b^{3}(z) a^{\prime}(z)}-\int \mu(z) \mathrm{d} z, \quad \omega=\frac{i b^{2} \mathrm{~d} a}{a}
$$

In section 8.2 it will be shown how the formula $\mathbf{I w a}_{a, b}$ is encoded with Mathematica. Also, the results of the above for the Bryant data $g, \omega$, the natural parameter and the null curvature of $\mathbf{I w} \mathbf{a}_{a, b}$ are computed there. The relevant Mathematica commands can be used to derive the following purely algebraic representation formula.

In order to derive a second integral formula let $\mathrm{F}=\left(\begin{array}{cc}A & B \\ C & D\end{array}\right)$ be a solution of $\mathrm{dF}=\mathrm{F} \mathcal{N}$, where $\mathcal{N}=\left(\begin{array}{cc}g & -g^{2} \\ 1 & -g\end{array}\right) \omega$ and $g$ and $\omega$ are a meromorphic function and a meromorphic 1-form respectively on a Riemannian surface $\Sigma$. A second integral representation formula is obtained by expressing the Bryant data $g$ and $\omega$ in terms of the functions $A, B$. Rewrite $\mathrm{dF}=\mathrm{F} \mathcal{N}$ as

$$
\left(\begin{array}{ll}
\mathrm{d} A & \mathrm{~d} B \\
\mathrm{~d} C & \mathrm{~d} D
\end{array}\right)=\left(\begin{array}{cc}
A & B \\
C & D
\end{array}\right) \mathcal{N}=\left(\begin{array}{cc}
A g+B & -g(A g+B) \\
C g+D & -g(C g+D)
\end{array}\right) \omega .
$$

We infer from (4.5) that

$$
g=-\frac{\mathrm{d} B}{\mathrm{~d} A}=-\frac{\mathrm{d} D}{\mathrm{~d} C} \quad \text { and } \quad \omega=A \mathrm{~d} C-C \mathrm{~d} A .
$$

Altogether, a list of various expressions for $\omega$ can be derived, of which we show the following samples:

$$
\omega=A \mathrm{~d} C-C \mathrm{~d} A=\frac{\mathrm{d} A^{2}}{B \mathrm{~d} A-A \mathrm{~d} B}=\frac{\mathrm{d} C^{2}}{D \mathrm{~d} C-C \mathrm{~d} D}=\frac{\mathrm{d} A \mathrm{~d} C}{D \mathrm{~d} A-C \mathrm{~d} B} .
$$


Considering now the functions $A$ and $B$ as independent variables, replacing $g=-\mathrm{d} B / \mathrm{d} A$ and $\omega=A \mathrm{~d} C-C \mathrm{~d} A$ in $\mathcal{N}$, we see that the vector $(A, B)$ is a solution of the system $(\mathrm{d} A, \mathrm{~d} B)=(A, B) \mathbf{B}_{g, \omega}$. A second linearly independent solution is obtained by the reduction of order of d'Alembert (see for instance [12]). This gives the following integral representation formula:

$$
\mathbf{B}_{g, \omega}=\left(\begin{array}{c|c}
A & B \\
\hline-A \int \alpha & \frac{1}{A}-B \int \alpha
\end{array}\right), \text { where } \alpha=\frac{\mathrm{d} A^{2}}{A^{2}(A \mathrm{~d} B-B \mathrm{~d} A)} .
$$

We simplify the 1-form $\alpha$ by substituting

$$
a=-1 / A \text { and } b=-B / A
$$

to obtain the simpler expression

$$
\mathbf{B}_{g, \omega}=\operatorname{Mod}_{a, b}=\frac{1}{a}\left(\begin{array}{c|c}
-1 & -b \\
\hline \int \beta & b \int \beta-a^{2}
\end{array}\right), \quad \text { where } \beta=\frac{\mathrm{d} a^{2}}{\mathrm{~d} b} .
$$

Proposition 4.1. Let $a, b$ be meromorphic functions on a Riemannian surface $\Sigma$ such that the differential $\beta$ of (4.8) can be integrated, i.e., there exist a meromorphic function $\mathbf{f}$ on $\Sigma$ such that $\mathrm{d} \mathbf{f}=\beta$. The formula (4.8) defines an operator $(a, b) \rightarrow \mathbf{M o d}_{a, b}$ mapping pairs of meromorphic functions on $\Sigma$ to null curves in $\mathrm{Sl}(2, \mathbb{C})$ whose Bryant data are

$$
g=-\frac{a \mathrm{~d} b-b \mathrm{~d} a}{\mathrm{~d} a}, \quad \omega=\frac{\mathrm{d} a^{2}}{a^{2} \mathrm{~d} b} .
$$

In terms of a local coordinate $z$ on $\Sigma$, the derivative of the natural parameter of the curve $\operatorname{Mod}_{a, b}$ is obtained by

$$
\left(\pi^{\prime}(z)\right)^{2}=\frac{b^{\prime}(z) a^{\prime \prime}(z)-a^{\prime}(z) b^{\prime \prime}(z)}{a(z) b^{\prime}(z)}
$$

This formula for $\pi^{\prime}$ follows from (4.9) and (3.9). One can obtain it also independently using the parameterization $\mathbf{x}$ of $\mathrm{Sl}(2, \mathbb{C})$ and the Christoffel symbols (2.6). As a consequence of this the natural parametrization of $\mathbf{M o d}_{a, b}$ is obtained if we choose $b_{\text {can }}(z)=\int a^{\prime} e^{-\int a / a^{\prime} \mathrm{d} z} \mathrm{~d} z$. For the null curvature $\kappa(z)$ of $\mathbf{M o d}_{a, b}$ a large differential expression in $a$ and $b$ is obtained, which becomes simpler in natural parametrization $\pi(z)$, using the function $b_{\text {can }}$ :

$$
\kappa(\pi)=\frac{8 a(\pi) a^{\prime 2}(\pi) a^{\prime \prime}(\pi)+a^{2}(\pi)\left(a^{\prime \prime 2}(\pi)-2 a^{\prime}(\pi) a^{(3)}(\pi)\right)-a^{4}(\pi)-8 a^{\prime 4}(\pi)}{a^{2}(\pi) a^{\prime 2}(\pi)} .
$$

Let us note that given an arbitrary function $\pi_{0}(z)$ as prospective infinitesimal natural parameter, the differential equation $\pi_{0}^{2}(z)=\frac{b^{\prime}(z) a^{\prime \prime}(z)-a^{\prime}(z) b^{\prime \prime}(z)}{a(z) b^{\prime}(z)}$ can be solved for $b(z)$ by integrations. Inserting the resulting expression in (4.8) gives the following integral representation formula for null curves with prescribed natural parameter. 


$$
\left\{\begin{array}{l}
\operatorname{Mod}_{a, \pi_{0}}=\frac{1}{a}\left(\begin{array}{cc}
-1 & -\nu_{0} \\
\nu_{2} & \nu_{0} \nu_{2}-a^{2}
\end{array}\right), \text { where } \nu_{0}(z)=\int_{0}^{z} e^{\mu(t)} \mathrm{d} t \\
\nu_{2}(z)=\int_{0}^{z} a^{\prime 2}(t) e^{\mu(t)} \mathrm{d} t, \quad \mu(t)=\int \frac{a^{\prime \prime}(t)-a(t) \pi_{0}(t)}{a^{\prime}(t)} \mathrm{d} t
\end{array}\right.
$$

5. A family of algebraic representation formulas for null curves in $\operatorname{Sl}(2, \mathbb{C})$. One can try to remove integration from any representation formula like (1.1), (4.3), or (4.8) by replacing integrands by differential expressions in other functions such that the integrals become explicit. So, for instance, replacing in the Weierstraß formula (1.2) the differential form $\omega$ by $g^{(3)}(z)$, where $g(z)$ is the local representative of $\mathbf{g}$ with respect to a local coordinate $z$ on $\Sigma$, gives integrals that can be made explicit by partial integration. A more refined and flexible method turning the Weierstraß formula into an algebraic one was studied in our paper [7], where we introduced differential operators $\mathbf{P}_{n}(\mathbf{g}, \mathbf{h})$, $(n=3,4, \ldots)$, mapping pairs $(\mathbf{g}, \mathbf{h})$ consisting of a meromorphic function $\mathbf{g}$ and a meromorphic vector field $\mathbf{h}$ on a Riemannian surface $\Sigma$ to exact forms $\omega$ on $\Sigma$. Moreover, also $\mathbf{g} \omega, \mathbf{g}^{2} \omega, \ldots, \mathbf{g}^{n-1} \omega$ are exact and functions $\mathbf{G}_{n, k}(\mathbf{g}, \mathbf{h})$ such that $\mathrm{d} \mathbf{G}_{n, k}(\mathbf{g}, \mathbf{h})=\mathbf{g}^{k} \omega$, $(k=0,1,2, \ldots, n-1)$, can be expressed as linear combinations of the operators $\mathbf{P}_{n}(\mathbf{g}, \mathbf{h})$, as will be explained below.

Replacing in (1.2) $\omega, \mathbf{g} \omega, \mathbf{g}^{2} \omega$ by $\mathrm{d} \mathbf{G}_{n, 0}(\mathbf{g}, \mathbf{h}), \mathrm{d} \mathbf{G}_{n, 1}(\mathbf{g}, \mathbf{h}), \mathrm{d} \mathbf{G}_{n, 2}(\mathbf{g}, \mathbf{h})$, respectively, yields an algebraic representation formula. It is shown that the operator $\mathbf{V}_{n}$ can be inverted without integrations by elementary operations and that it is $\mathrm{Sl}(2, \mathbb{C})$-invariant. A slightly different version of $\mathbf{V}_{3}$ was introduced in ([9]) by another method turning Bryant's formula into an algebraic one. We call it the Bianchi-Small formula in the next section (see formula (6.1)).

We are now going to show how the operators $\mathbf{G}_{n, k}$ of [7] can be applied to turn (4.8) into an algebraic formula. Let $\Sigma$ be a connected Riemannian surface, $\mathcal{A}_{\Sigma}$ the set of non-constant meromorphic functions, $\mathcal{X}_{\Sigma}$ the space of meromorphic vector fields, $\mathcal{L}_{\Sigma}$ the space of meromorphic 1-forms, and

$$
\left(\mathcal{A}_{\Sigma} \times \mathcal{L}_{\Sigma}\right)_{n}^{*}=\left\{(\mathbf{f}, \omega) \in \mathcal{A}_{\Sigma} \times \mathcal{L}_{\Sigma} \mid \omega, \mathbf{f} \omega, \mathbf{f}^{2} \omega \ldots, \mathbf{f}^{n-1} \omega \text { are exact }\right\} .
$$

A sequence $\mathbf{P}_{n}: \mathcal{A}_{\Sigma} \times \mathcal{X}_{\Sigma} \rightarrow \mathcal{L}_{\Sigma}$ of nonlinear differential operators such that $\left(\mathbf{f}, \mathbf{P}_{n}(\mathbf{f}, \mathbf{h})\right) \in\left(\mathcal{A}_{\Sigma} \times \mathcal{L}_{\Sigma}\right)_{n}^{*}$ for $n \geq 3$ and $(\mathbf{f}, \mathbf{h}) \in \mathcal{A}_{\Sigma} \times \mathcal{X}_{\Sigma}$ is given by:

$$
\mathbf{P}_{0}(\mathbf{f}, \mathbf{h})=\langle\mathrm{d} \mathbf{f}, \mathbf{h}\rangle \mathrm{d} \mathbf{f} \quad \text { and } \quad \mathbf{P}_{n}(\mathbf{f}, \mathbf{h})=\mathrm{d}\left(\frac{\mathbf{P}_{n-1}(\mathbf{f}, \mathbf{h})}{\mathrm{d} \mathbf{f}}\right) .
$$

Showing that $\left(\mathbf{f}, \mathbf{P}_{n}(\mathbf{f}, \mathbf{h})\right) \in\left(\mathcal{A}_{\Sigma} \times \mathcal{L}_{\Sigma}\right)_{n}^{*}$ for all $(\mathbf{f}, \mathbf{h}) \in \mathcal{A}_{\Sigma} \times \mathcal{X}_{\Sigma}$ is a simple induction argument (see [7]). The first three members of the sequence $\mathbf{P}_{n}(f, h)$ are

$$
\left\{\begin{array}{l}
\mathbf{P}_{1}=f^{\prime} h^{\prime}+h f^{\prime \prime}, \\
\mathbf{P}_{2}=\frac{f^{\prime} h^{\prime} f^{\prime \prime}-h f^{\prime \prime 2}+f^{\prime 2} h^{\prime \prime}+h f^{\prime} f^{(3)}}{f^{\prime 2}}, \text { and } \\
\mathbf{P}_{3}=-\frac{3 f^{\prime} h^{\prime} f^{\prime \prime 2}+3 h f^{\prime \prime 3}+2 f^{\prime 2} h^{\prime} f^{(3)} 4 h f^{\prime} f^{\prime \prime} f^{(3)}+f^{\prime 3} h^{(3)}+h f^{\prime 2} f^{(4)}}{f^{\prime 4}} .
\end{array}\right.
$$

In the local setting, the operators $h \rightarrow \mathbf{P}_{n}(f, h)$ can be inverted by integration: 
Proposition 5.1. If $\omega=\mathbf{P}_{n}(f, h)$ then

$$
h=\frac{1}{\mathrm{~d} f}\left(\frac{1}{(n-1) !} \sum_{i=0}^{n-1}\left\{(-1)^{i}\left(\begin{array}{c}
n \\
i
\end{array}\right) f^{n-i} \int f^{i} \omega\right\}+\sum_{i=0}^{n-1} c_{i} f^{i}\right),
$$

where $c_{0}, c_{1}, \ldots, c_{n-1}$ are constants of integration.

In addition to this property, the integrals of $\mathbf{f}^{i} \mathbf{P}_{n}(\mathbf{f}, \mathbf{h})$ can be expressed explicitly in terms of $\mathbf{f}$ and $\mathbf{P}_{n-k-1}(\mathbf{f}, \mathbf{h}), \mathbf{P}_{n-k}(\mathbf{f}, \mathbf{h}), \ldots, \mathbf{P}_{n-1}(\mathbf{f}, \mathbf{h})$. To this end we define a double sequence $\mathbf{G}_{k, n}: \mathcal{A}_{\Sigma} \times \mathcal{X}_{\Sigma} \rightarrow \mathcal{A}_{\Sigma}, n=3,4,5, \ldots, 0 \leq k<n$, of nonlinear differential operators recursively by

$$
\left\{\begin{array}{l}
\mathbf{G}_{0,1}(\mathbf{f}, \mathbf{h})=\langle\mathrm{d} \mathbf{f}, \mathbf{h}\rangle, \quad \mathbf{G}_{0, n}(\mathbf{f}, \mathbf{h})=\frac{\mathbf{P}_{n-1}(\mathbf{f}, \mathbf{h})}{\mathrm{d} \mathbf{f}} \text { and } \\
\mathbf{G}_{k, n}(\mathbf{f}, \mathbf{h})=\mathbf{f}^{k} \frac{\mathbf{P}_{n-1}(\mathbf{f}, \mathbf{h})}{\mathrm{d} \mathbf{f}}-k \mathbf{G}_{k-1, n-1}(\mathbf{f}, \mathbf{h}) .
\end{array}\right.
$$

Then, $\mathrm{d} \mathbf{G}_{k, n}(\mathbf{f}, \mathbf{h})=\mathbf{f}^{k} \mathbf{P}_{n}(\mathbf{f}, \mathbf{h})$, as can be proved by induction. From the recursion rule (5.2) the following explicit expression of $\mathbf{G}_{k, n}$ is derived:

$$
\mathbf{G}_{k, n}(\mathbf{f}, \mathbf{h})=\sum_{j=0}^{k}(-1)^{k-j} \frac{k !}{j !} \mathbf{f}^{j} \frac{\mathbf{P}_{j+n-k-1}(\mathbf{f}, \mathbf{h})}{\mathrm{d} \mathbf{f}} .
$$

Local expressions of $\mathbf{G}_{k, 3}(f, h)$ for $k=0,1,2$ are

$$
\begin{aligned}
\mathbf{G}_{0,3} & =\frac{1}{f^{\prime 3}}\left(f^{\prime} h^{\prime} f^{\prime \prime}-h f^{\prime \prime 2}+f^{\prime 2} h^{\prime \prime}+h f^{\prime} f^{(3)}\right), \\
\mathbf{G}_{1,3}= & \frac{1}{f^{\prime 3}}\left(f f^{\prime 2} h^{\prime \prime}+f h f^{\prime} f^{(3)}-f^{\prime 3} h^{\prime} h f^{2} f^{\prime \prime}+f f^{\prime} h^{\prime} f^{\prime \prime}-f h f^{\prime \prime 2}\right), \\
\mathbf{G}_{2,3}= & \frac{1}{f^{\prime 3}}\left(2 h f^{\prime 4}-2 f f^{\prime 3} h^{\prime}-2 f h f^{\prime 2} f^{\prime \prime}\right. \\
& \left.\quad+f^{2} f^{\prime} h^{\prime} f^{\prime \prime}-f^{2} h f^{\prime \prime 2}+f^{2} f^{\prime 2} h^{\prime \prime}+f^{2} h f^{\prime} f^{(3)}\right) .
\end{aligned}
$$

Another useful property of the operators $\mathbf{G}_{k, n}$ is the following:

THEOREM 5.1. For any natural numbers $n, k, l$ with

$$
n>2, \quad k \in\{0,1, \ldots, n-1\} \quad \text { and } \quad l \in\{0,1, \ldots, \operatorname{Min}\{k, n-k-1\}\}
$$

we have

$$
\mathrm{d} \mathbf{G}_{k, n} \mathrm{~d} \mathbf{G}_{k, n}=\mathrm{d} \mathbf{G}_{k-l, n} \mathrm{~d} \mathbf{G}_{k+l, n} .
$$

The proof follows trivially from the fact that $\mathrm{d}_{\mathbf{G}_{k, n}}(\mathbf{f}, \mathbf{h})=\mathbf{f}^{k} \mathbf{P}_{n}(\mathbf{f}, \mathbf{h})$ and $\mathrm{d} \mathbf{G}_{k \pm l, n}(\mathbf{f}, \mathbf{h})=\mathbf{f}^{k \pm l} \mathbf{P}_{n}(\mathbf{f}, \mathbf{h})$.

By means of the operators $\mathrm{d} \mathbf{G}_{k, n}$, the integral $\int \beta=\int \mathrm{d} a^{2} / \mathrm{d} b$ occurring in (4.8) can be made explicit. Putting $a=\mathbf{G}_{k, n}(\mathbf{f}, \mathbf{h})$ and $b=\mathbf{G}_{k-l, n}$ gives $\int \mathrm{d} a^{2} / \mathrm{d} b=\mathbf{G}_{k+l, n}$ by (5.4).

Corollary 5.1. Let $n, k, l$ be integers as above. For any Riemannian surface $\Sigma$ the operator $\mathbf{M}^{(n, k, l)}$, defined below, maps $\mathcal{A}_{\Sigma} \times \mathcal{X}_{\Sigma}$ into the space $\mathbf{N}_{\Sigma}$ of null curves.

$$
(\mathbf{f}, \mathbf{h}) \in \mathcal{A}_{\Sigma} \times \mathcal{X}_{\Sigma} \rightarrow \mathbf{M}_{\mathbf{f}, \mathbf{h}}^{(n, k, l)}=\frac{1}{\mathbf{G}_{k, n}}\left(\begin{array}{c|c}
-1 & -\mathbf{G}_{k-l, n} \\
\hline \mathbf{G}_{k+l, n} & \mathbf{G}_{k-l, n} \mathbf{G}_{k+l, n}-\mathbf{G}_{k, n}^{2}
\end{array}\right)
$$


For the following let us confine ourselves to the case $l=1$ and denote $\mathbf{M}_{\mathbf{f}, \mathbf{h}}^{(n, k)}=$ $\mathbf{M}_{\mathbf{f}, \mathbf{h}}^{(n, k, 1)}$. The operator $\mathbf{M}_{\mathbf{f}, \mathbf{h}}^{(n, k)}$ can be inverted by integrations in the following way:

Proposition 5.2. Let $\mathrm{F}$ be a null curve in $\mathrm{Sl}(2, \mathbb{C})$ and denote the entries of its first row by $A=\mathrm{F}_{1,1}$ and $B=\mathrm{F}_{1,2}$. Then a function $\mathbf{f} \in \mathcal{A}_{\Sigma}$ and a vector field $\mathbf{h} \in \mathcal{X}_{\Sigma}$ such that $\mathrm{F}=\mathbf{M}_{\mathbf{f}, \mathbf{h}}^{(n, k)}$ are obtained as

$$
\mathbf{f}=\frac{\mathrm{d} A}{B \mathrm{~d} A-A \mathrm{~d} B}
$$

and

$$
\mathbf{h}=\frac{1}{\mathrm{~d} \mathbf{f}} \frac{1}{(n-1) !} \sum_{i=0}^{n-1}\left\{(-1)^{i}\left(\begin{array}{c}
n \\
i
\end{array}\right) \mathbf{f}^{n-i} \int \mathbf{f}^{i} \omega\right\}+\frac{1}{\mathrm{~d} \mathbf{f}} \sum_{i=0}^{n-1} c_{i} \mathbf{f}^{i}, \text { where } \omega=\frac{1}{\mathbf{f}^{k}} \mathrm{~d}\left(\frac{-1}{A}\right) .
$$

Proof. Start from (5.5) with $A=-1 / \mathbf{G}_{k, n}(\mathbf{f}, \mathbf{h}), B=-\mathbf{G}_{k-1, n}(\mathbf{f}, \mathbf{h}) / \mathbf{G}_{k, n}(\mathbf{f}, \mathbf{h})$. We obtain

$$
\left\{\begin{array}{c}
\mathrm{d}(-1 / A)=\mathrm{d} \mathbf{G}_{k, n}(\mathbf{f}, \mathbf{h})=\mathbf{f}^{k} \mathbf{P}_{n}(\mathbf{f}, \mathbf{h}) \text { and } \\
\mathrm{d}(-B / A)=\mathrm{d} \mathbf{G}_{k-1, n}(\mathbf{f}, \mathbf{h})=\mathbf{f}^{k-1} \mathbf{P}_{n+1}(\mathbf{f}, \mathbf{h})
\end{array}\right.
$$

Therefore:

$$
\frac{\mathrm{d}(-1 / A)}{\mathrm{d}(-B / A)}=\frac{\mathrm{d} A}{B \mathrm{~d} A-A \mathrm{~d} B}=\frac{\mathbf{f}^{k} \mathbf{P}_{n}(\mathbf{f}, \mathbf{h})}{\mathbf{f}^{k-1} \mathbf{P}_{n}(\mathbf{f}, \mathbf{h})}=\mathbf{f} .
$$

This gives $\mathbf{f}$ in terms of $A$ and $B$. In order to obtain $\mathbf{h}$ it is sufficient to divide the first of the equations (5.6) by $\mathbf{f}^{k}$. One obtains the equation $\omega=\mathbf{f}^{-k} \mathrm{~d}(-1 / A)=\mathbf{P}_{n}(\mathbf{f}, \mathbf{h})$. The inversion formula of proposition (5.1) gives an expression of $\mathbf{h}$ in terms of $\omega$ as announced.

In a similar way, the Bryant data of $\mathbf{M}_{a, b}^{(n, k, l)}$ can be computed using (4.6):

Proposition 5.3. Let $\mathbf{f}$ and $\mathbf{h}$ be a meromorphic function and a meromorphic vector field on $\Sigma$ respectively and let a null curve $\mathrm{F}: \Sigma \rightarrow \mathrm{Sl}(2, \mathbb{C})$ be given as $\mathrm{F}(z)=\mathbf{M}_{\mathbf{f}, \mathbf{h}}^{(n, k)}(z)$ for $n-1>k>0$. Then $\mathrm{F}(z)=\mathbf{B}_{\mathbf{g}, \omega}(z)$, where the Bryant data $(\mathbf{g}, \omega)$ of $\mathrm{F}(z)$ are

$$
\mathbf{g}=\mathbf{f}^{-l} \mathbf{G}_{k, n}(\mathbf{f}, \mathbf{h})-\mathbf{G}_{k-l, n}(\mathbf{f}, \mathbf{h}), \quad \omega=-\frac{\mathbf{f}^{k+l} \mathbf{P}_{n}(\mathbf{f}, \mathbf{h})}{\mathbf{G}_{k, n}^{2}(\mathbf{f}, \mathbf{h})} .
$$

Equation (5.7) gives d $\mathbf{g}=-l \mathbf{f}^{-l-1} \mathbf{G}_{k, n}(\mathbf{f}, \mathbf{h}) \mathrm{d} \mathbf{f}$. Therefore, the squared infinitesimal natural parameter is

$$
\omega \mathrm{d} \mathbf{g}=\frac{l \mathbf{f}^{k-1} \mathbf{P}_{n}(\mathbf{f}, \mathbf{h}) \mathrm{d} \mathbf{f}}{\mathbf{G}_{k, n}(\mathbf{f}, \mathbf{h})} .
$$

This is also the Hopf differential of the cmc-1-surface corresponding to $\mathbf{B}_{\mathbf{g}, \omega}(z)$.

6. An invariant version of the representation formula of A. J. Small. In this section we consider a modified version of an algebraic representation formula for null curves in $\mathrm{Sl}(2, \mathbb{C})$ that will be called the Bianchi-Small formula here. It was developed by A. J. Small in [11] with techniques of algebraic geometry. However, in implicit form it can be found in the classical book [2] of L. Bianchi where its derivation was based on geometric ideas concerning congruences of spheres and the rolling of pairs of isometric 
surfaces in $\mathbb{R}^{3}$ on each other (see also [10]). The following algebraic formula is quoted from [11]:

$$
\mathbf{B S}_{f}=\frac{1}{2\left(f^{\prime}\right)^{3 / 2}}\left(\begin{array}{cc}
2 f^{\prime 2}-f f^{\prime \prime} & f\left(2 f^{\prime}+z f^{\prime \prime}\right)-2 z f^{\prime 2} \\
-f^{\prime \prime} & 2 f^{\prime}+z f^{\prime \prime}
\end{array}\right) .
$$

Its Bryant data are $g(z)=z$ and $\omega(z)=(\mathcal{S}(f)(z))^{2} / 4$, where $\mathcal{S}(f)(z)$ is the Schwarzian derivative of $f$, i.e., the system (1.1) becomes

$$
\frac{\mathrm{d}}{\mathrm{d} z} \mathbf{B S}_{f}(z)=\frac{3 f^{\prime \prime 2}-2 f^{\prime} f^{(3)}}{4 f^{\prime 2}} \mathbf{B S}_{f}(z)\left(\begin{array}{cc}
z & -z^{2} \\
1 & -z
\end{array}\right) \text {. }
$$

Let $\Sigma$ be a Riemannian surface. In order to turn $\mathbf{B S}_{f}$ into a globally defined differential operator $\mathbf{B S}_{f}: \mathcal{A}_{\Sigma} \times \mathcal{A}_{\Sigma} \rightarrow \mathbf{N}_{\Sigma}$ mapping pairs (f, g) of meromorphic functions on $\Sigma$ to null curves in $\mathrm{Sl}(2, \mathbb{C})$ let us define a partial operation of muliplication on the set $\mathbf{N}_{\Sigma}$. Let $g$ and $\omega$ be a meromorphic function and a meromorphic 1-form on $\mathbb{C}$ respectively. Here we denote by $\mathrm{F}_{g, \omega}$ the null curve with Bryant data $(g, \omega)$, and by $A_{g, \omega}, B_{g, \omega}, C_{g, \omega}, D_{g, \omega}$ the entries of the matrix $\mathrm{F}_{g, \omega}$, i.e.,

$$
\mathrm{F}_{g, \omega}=\left(\begin{array}{cc}
A_{g, \omega} & B_{g, \omega} \\
C_{g, \omega} & D_{g, \omega}
\end{array}\right) \text { with } \mathrm{F}_{g, \omega}^{\prime}=\mathrm{F}_{g, \omega} X_{g, \omega} \text { and } X_{g, \omega}=\left(\begin{array}{cc}
g & -g^{2} \\
1 & -g
\end{array}\right) \omega .
$$

Two null curves $\mathrm{F}_{g, \omega_{1}}$ and $\mathrm{F}_{g, \omega_{2}}$ whose pairs $\left(g, \omega_{1}\right),\left(g, \omega_{2}\right)$ of Bryant data agree in their first member can be combined as $\mathrm{F}_{g, \omega_{1}} \mathrm{~F}_{g, \omega_{2}}^{-1}$ to form a new null curve, namely

Proposition 6.1. For any meromorphic function $g$ and any meromorphic 1 -forms $\omega_{1}, \omega_{1}$ $\mathrm{F}_{g, \omega_{1}} \mathrm{~F}_{g, \omega_{2}}^{-1}=\mathrm{F}_{\widetilde{g}, \widetilde{\omega}}$ where

$$
\widetilde{\omega}=\left(\omega_{1}-\omega_{2}\right)\left(C_{g, \omega_{2}} g+D_{g, \omega_{2}}\right)^{2} \quad \text { and } \quad \widetilde{g}=\frac{A_{g, \omega_{2}} g+B_{g, \omega_{2}}}{C_{g, \omega_{2}} g+D_{g, \omega_{2}}} .
$$

Proof. Put $\mathrm{F}=\mathrm{F}_{g, \omega_{1}} \mathrm{~F}_{g, \omega_{2}}^{-1}$. From $\mathrm{dF}_{g, \omega_{1}}=\mathrm{F}_{g, \omega_{1}} X_{g, \omega_{1}}, \mathrm{dF}_{g, \omega_{2}}=\mathrm{F}_{g, \omega_{2}} X_{g, \omega_{2}}$ it follows that

$$
\begin{aligned}
\mathrm{F}^{-1} \mathrm{dF} & =\mathrm{F}_{g, \omega_{2}} \mathrm{~F}_{g, \omega_{1}}^{-1}\left(\mathrm{~F}_{g, \omega_{1}} X_{g, \omega_{1}} \mathrm{~F}_{g, \omega_{2}}^{-1}-\mathrm{F}_{g, \omega_{1}} \mathrm{~F}_{g, \omega_{2}}^{-1} \mathrm{~F}_{g, \omega_{2}} X_{g, \omega_{2}} \mathrm{~F}_{g, \omega_{2}}^{-1}\right) \\
& =\mathrm{F}_{g, \omega_{2}}\left(X_{g, \omega_{1}}-X_{g, \omega_{2}}\right) \mathrm{F}_{g, \omega_{2}}^{-1}=\mathrm{F}_{g, \omega_{2}} X_{g, \omega_{1}-\omega_{2}} \mathrm{~F}_{g, \omega_{2}}^{-1} .
\end{aligned}
$$

The last matrix is

$$
\begin{array}{r}
\left(\omega_{1}-\omega_{2}\right)\left(\begin{array}{cc}
A_{g, \omega_{2}} & B_{g, \omega_{2}} \\
C_{g, \omega_{2}} & D_{g, \omega_{2}}
\end{array}\right)\left(\begin{array}{cc}
g & -g^{2} \\
1 & -g
\end{array}\right)\left(\begin{array}{cc}
D_{g, \omega_{2}} & -B_{g, \omega_{2}} \\
-C_{g, \omega_{2}} & A_{g, \omega_{2}}
\end{array}\right) \\
=\left(\omega_{1}-\omega_{2}\right)\left(C_{g, \omega_{2}} g+D_{g, \omega_{2}}\right)^{2}\left(\begin{array}{cc}
\widetilde{g} & -\widetilde{g}^{2} \\
1 & -\widetilde{g}
\end{array}\right)=X_{\widetilde{g}, \widetilde{\omega}} . \mathbf{}
\end{array}
$$

The Bryant data of any two null curves of the form $\mathbf{B} \mathbf{S}_{f_{1}}$ and $\mathbf{B} \mathbf{S}_{f_{2}}$ agree in their first entries: $g_{1}(z)=z$. Therefore, a new algebraic representation formula, denoted by the same symbol but depending on two meromorphic functions, is obtained as

$$
\mathbf{B S}_{f, g}=\mathbf{B S}_{f} \mathbf{B S}_{g}^{-1} \text {. }
$$

A direct computation gives: 
Proposition 6.2. The derivative of $\mathbf{B S}_{f, g}$ is

$$
\frac{\mathrm{dBS}_{f, g}(z)}{\mathrm{d} z}=\frac{(\mathcal{S}(f)(z))^{2}-(\mathcal{S}(g)(z))^{2}}{\sqrt{f^{\prime}(z)} \sqrt{g^{\prime}(z)}}\left(\begin{array}{cc}
f(z) & -f(z) g(z) \\
1 & -g(z)
\end{array}\right) .
$$

Therefore, an inversion formula and the Bryant data of $\mathbf{B S}_{f, g}$ are obtained immediately.

Proposition 6.3. If

$$
\mathbf{B S}_{f, g}(z)=\left(\begin{array}{cc}
A & B \\
C & D
\end{array}\right) \text { then } f=\frac{\mathrm{d} A}{\mathrm{~d} C}, \quad g=-\frac{\mathrm{d} D}{\mathrm{~d} C} .
$$

$\mathbf{B S}_{f, g}$ can be expressed by the Bryant formula as $\mathbf{B S}_{f, g}=\mathbf{B}_{g, \omega}$, where

$$
\omega=\frac{(\mathcal{S}(f)(z))^{2}-(\mathcal{S}(g)(z))^{2}}{4 g^{\prime}(z)} .
$$

Since the difference of the squares of the Schwarzian derivatives of two meromorphic functions is a quadratic form, we conclude that if $\Sigma$ is a Riemannian surface and $\mathbf{f}, \mathbf{g}$ are two meromorphic functions on $\Sigma, f, g$ the local expressions of $\mathbf{f}, \mathbf{g}$ in some holomorphic chart then the operator $(\mathbf{f}, \mathbf{g}) \rightarrow \mathbf{B S}_{\mathbf{f}, \mathbf{g}}:=\mathbf{B S}_{f, g}$ is well defined on $\Sigma$ and yields an algebraic representation formula for null curves.

Proposition 6.4. Let $\Sigma$ be a Riemannian surface, $\mathbf{f}, \mathbf{g} \in \mathcal{A}_{\Sigma}$, and $f(z), g(z)$ local representatives of $\mathbf{f}, \mathbf{g}$ respectively. The quadratic differential $\mathrm{d} \pi^{2}$ of the squared infinitesimal parameter of $\mathbf{B S}_{\mathbf{f}, \mathbf{g}}$ is $\mathrm{d} \pi^{2}=\mathrm{d} \mathbf{g} \omega=\left((\mathcal{S}(f)(z))^{2}-(\mathcal{S}(g)(z))^{2}\right) / 4$. For the null curvature of $\mathbf{B S}_{\mathbf{f}, \mathbf{g}}$ we obtain with the help of Mathematica the following local expression:

$$
\kappa_{f, g}^{2}(z)=\frac{5 \Delta^{\prime}(z)^{2}}{4 \Delta(z)^{3}}-\frac{\Delta^{\prime \prime}(z)}{\Delta(z)^{2}}-\frac{(\mathcal{S}(g)(z))^{2}}{\Delta(z)}-2
$$

where $\Delta(z)=\left((\mathcal{S}(f)(z))^{2}-(\mathcal{S}(g)(z))^{2}\right) / 4$ is the local expression of $\mathrm{d} \pi^{2}$, i.e., $\mathrm{d} \pi^{2}=$ $\Delta(z) \mathrm{d} z^{2}$.

We note that (6.8) has global meaning by definition. It does not depend on the local variable $z$, i.e., if $w=\tau(z)$ is an arbitrary parameter transformation then $\kappa_{f \circ \tau, f \circ \tau}^{2}(z)=$ $\kappa_{f, g}^{2}(\tau(z))$. A Mathematica verification of this and derivation of (6.8) are given in section 8.2.

Occurrence of Schwarzian derivatives in (6.7) suggests that $\mathbf{B S}_{\mathbf{f}, \mathbf{g}}$ should be invariant with respect to the action of $\operatorname{Sl}(2, \mathbb{C})$ by fractional linear operations on functions $\mathbf{f}, \mathbf{g} \in$ $\mathcal{A}_{\Sigma}$. In fact, let $R=\left(\begin{array}{ll}a & b \\ c & d\end{array}\right)$ be an $\mathrm{Sl}(2, \mathbb{C})$-matrix and

$$
\widetilde{\mathbf{f}}=\frac{a \mathbf{f}+b}{c \mathbf{f}+d}, \quad \widetilde{\mathbf{g}}=\frac{a \mathbf{g}+b}{c \mathbf{g}+d} \text { and, consequently, d } \widetilde{\mathbf{g}}=(c \mathbf{g}+d)^{-2} \mathrm{~d} \mathbf{g} .
$$

A direct computation with (6.6) gives:

Proposition 6.5. If $R, \mathbf{f}, \mathbf{g}, \widetilde{\mathbf{f}}, \widetilde{\mathbf{g}}$ are as above then $\mathbf{B S}_{\widetilde{\mathbf{f}}, \widetilde{\mathbf{g}}}=R \mathbf{B S}_{\mathbf{f}, \mathbf{g}} R^{-1}$.

Finally, let us note that, up to sign, the operator $(\mathbf{f}, \mathbf{g}) \rightarrow \mathbf{B S}_{\mathbf{f}, \mathbf{g}}$ coincides with a representation formula of Small-type derived in [9] by elementary operations as 


$$
(g, G) \rightarrow F=\left(\begin{array}{cc}
G p-a & G q-b \\
p & q
\end{array}\right), a=\sqrt{\frac{d G}{d g}}, b=-g a, p=\frac{d a}{d G}, q=\frac{d b}{d G} .
$$

Conversely, given a null curve as above, $g$ and $G$ are reobtained from $F$ as the hyperbolic Gauss map $G=d A / d C=d B / d D$ and the secondary Gauss map $g=-d B / d A=$ $-d D / d C$.

7. Mathematica implementations. From any Mathematica window the package $\mathrm{cmc1}$. $\mathrm{m}$ can be loaded by typing $<<\mathrm{cmc1}$. $\mathrm{m}$, provided it is contained in one of the directories specified in the path variable \$Path of the Mathematica installation, for instance in the actual working directory. All commands come with a usage information, accessed by typing ?command and telling something on functionality, meaning and format (functions, matrices, numbers, etc.) and arrangement of the arguments.

Bryant data, squared infinitesimal natural parameter and null curvature of an arbitrary null curve $\mathrm{F}$ are obtained by the commands nullcurveToBryantdata, nullcurveCanParameterPrime and nullcurveCurvature respectively. The first one is a Mathematica version of formulas like (3.7) or (4.6). The latter are obtained by inverting the operator $\operatorname{Mod}_{a, b}$ of (4.8). By (4.7), functions $a$ and $b$ such that $\mathbf{M o d}_{a, b}$ is the given null curve $\mathrm{F}$ are $a=-1 / A$ and $b=-B / A$ where $A=\mathrm{F}_{1,1}$ and $B=-\mathrm{F}_{1,2} / \mathrm{F}_{1,1}$. Having $a$ and $b, \pi^{\prime 2}$ and $\kappa$ are obtained with Mathematica versions of the formulas (4.10) and the general, more involved case of (4.11) related to arbitrary parametrization of $\mathrm{F}$.

7.1. Null curves of constant curvature. Curves with constant curvature are analogues of helices in $\mathbb{R}^{3}$. The command S12CFrameModified [a,b] [z] of cmc1.m is the Mathematica counterpart of the integral representation formula $\operatorname{Mod}_{a, b}(z)$ (see (4.8)). Choosing $a(z)=$ $e^{m z}$ and $b(z)=e^{n z}$ as arguments for this operator gives null curves of constant curvature. Null curves with prescribed values $\pi^{\prime}(z)=a$ and $\kappa=b, a, b \in \mathbb{C}$, are obtained for

$$
m=\frac{a \sqrt{\sqrt{b^{2}-4}-b}}{\sqrt{2}}, \quad n=\frac{a\left(\sqrt{b^{2}-4}-b-2\right)}{\sqrt{2} \sqrt{\sqrt{b^{2}-4}-b}} .
$$

The corresponding curve is

$$
\mathrm{F}_{a, b}(z)=\operatorname{Mod}_{e^{m z}, e^{n z}}(z)=\left(\begin{array}{cc}
-e^{-(m z)} & -e^{(-m+n) z} \\
\frac{e^{m z-n z} m^{2}}{2 m n-n^{2}} & \frac{e^{m z}(m-n)^{2}}{(2 m-n) n}
\end{array}\right) .
$$

This can be seen by typing

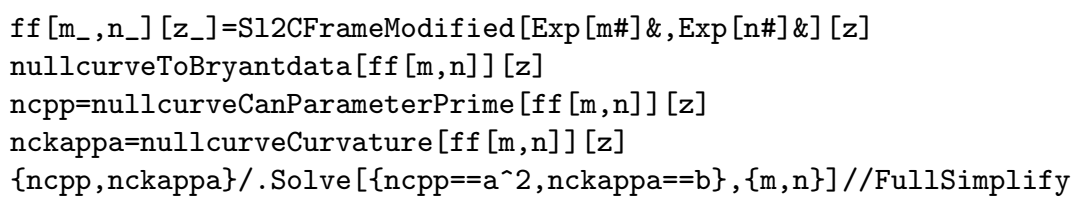




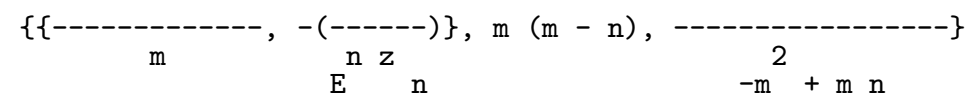

$\left\{\left\{\mathrm{a}^{2}, \mathrm{~b}\right\},\left\{\mathrm{a}^{2}, \mathrm{~b}\right\},\left\{\mathrm{a}^{2}, \mathrm{~b}\right\},\left\{\mathrm{a}^{2}, \mathrm{~b}\right\}\right\}$

We show plots of the corresponding cmc-1-surfaces in the ball model of $\mathbb{H}^{3}$ for several values of $a$ and $b$ :
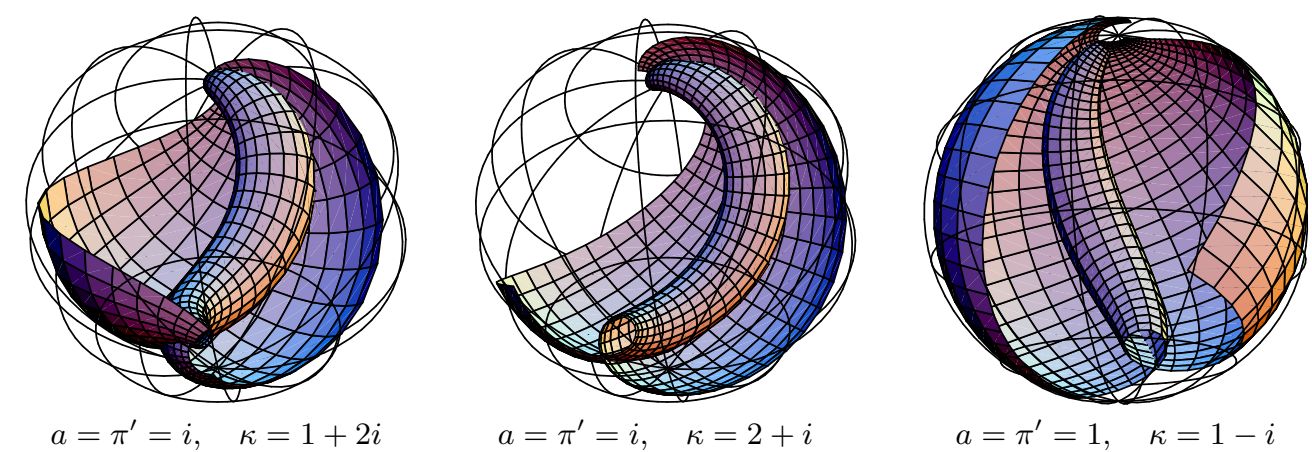

Curves with constant curvature can also be produced with Bryant's formula. Let us put $g(z)=z^{2}, \omega(z)=a z$. The null curve $\mathbf{B}_{g, \omega}$ is obtained with the command ff $\left[a_{-}\right]\left[z_{-}\right]=$Sl2CFrameBR2 [\#^2\&, a\#\&] [z]//FullSimplify

producing the response

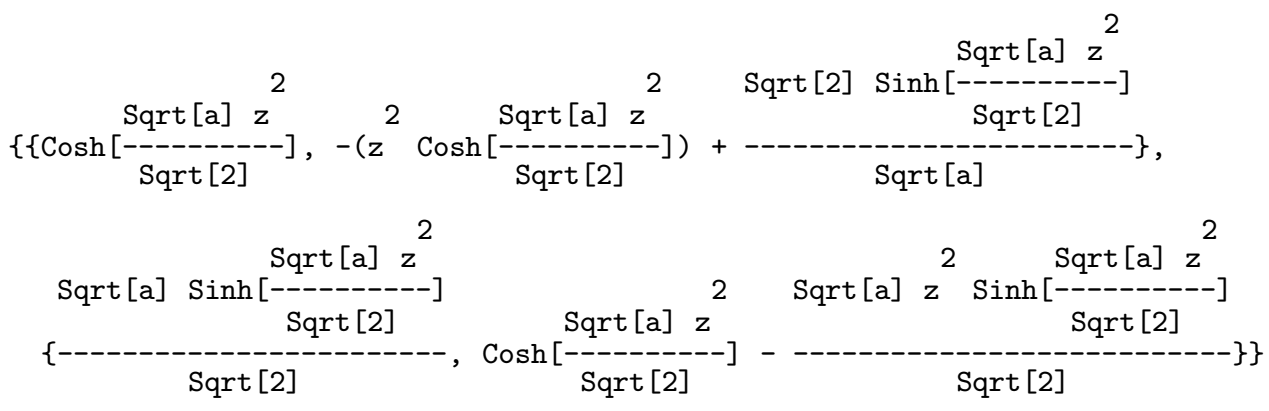

The natural parameter and the null curvature of this curve are:

$\{$ nullcurveCanParameterPrime $[f f[a]][z]$, nullcurveCurvature $[f f[a]][z]\}$

$\left\{2 \mathrm{a}^{2},-2\right\}$

By theorem 3.1, since for $b=-2$ the curvatures agree this curve must be a right translate of a reparametrization of the curve $\mathrm{F}_{a, b}=\operatorname{Mod}_{e^{m} z, e^{n z}}$ given in 7.1.

By proposition 6.1 two curves $\mathbf{B}_{z^{2}, a z}$ and $\mathbf{B}_{z^{2}, b z}$ can be combined to give a new null curve

$$
\mathrm{F}(z)=\mathbf{B}_{z^{2}, a z}\left(\mathbf{B}_{z^{2}, b z}\right)^{-1}=\left(\begin{array}{cc}
c_{a} c_{b}-\frac{s_{a} s_{b} \sqrt{b}}{\sqrt{a}} & \sqrt{2}\left(\frac{c_{b} s_{a}}{\sqrt{a}}-\frac{c_{a} s_{b}}{\sqrt{b}}\right) \\
\frac{c_{b} s_{a} \sqrt{a}-c_{a} s_{b} \sqrt{b}}{\sqrt{2}} & c_{a} c_{b}-\frac{s_{a} s_{b} \sqrt{a}}{\sqrt{b}}
\end{array}\right)
$$


where $c_{b}=\cosh \left(\sqrt{b} z^{2} / \sqrt{2}\right), c_{a}=\cosh \left(\sqrt{a} z^{2} / \sqrt{2}\right), s_{b}=\sinh \left(\sqrt{b} z^{2} / \sqrt{2}\right), s_{a}=$ $\sinh \left(\sqrt{a} z^{2} / \sqrt{2}\right)$. This curve has constant infinitesimal natural parameter and constant null curvature, namely, its Bryant data, infinitesimal natural parameter and curvature are the following:

$f f\left[z_{-}\right]=f f[a][z]$.Inverse $[f f[b][z]] / / F u l l$ Simplify;

nullcurveToBryantdata $[f f][z]$;

\{nullcurveCanParameterPrime [ff] [z]//Sqrt, nullcurveCurvature [ff] [z]\}

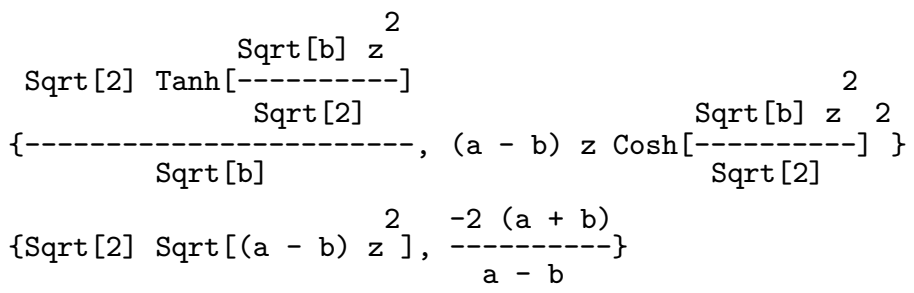

7.2. Implementations of Bryant's formula and cmc-1-cousins of minimal surfaces. Trying to attack the system (1.1) directly with Mathematica command NDSolve will be unsuccessful even for simplest input data $(g, \omega)$. However, NDSolve is more successful if fed with Riccati or ordinary linear equations. The package $\mathrm{cmc1}$. $\mathrm{m}$ contains two programs S12CFrameBR [g, omega] [z] and S12CFrameBR1 [g, omega] [c1, c2] [z] that reduce the integration of (1.1) to that of two (different) Riccati-equations and several integrations. A third program S12CFrameBR2 [g, om] [c1, c2] [z] tries to find algebraic solutions by reducing the system to a homogeneous second order linear equation. Often, the output is highly involved, containing huge expressions in special functions. It should be taken with some care and checked with other methods. We could observe that S12CFrameBR2 in Mathematica version 4.1 produced incorrect results and found that the error was caused by fake solutions that NDSolve returned to differential equations $x^{2} y^{\prime \prime}+a x y^{\prime}+b y=0$ of Euler type with integer coefficients $a, b$.

Let $(g, \omega)$ be the pair of Bryant data of a null curve $\mathrm{F}(z)=\mathbf{B}_{g, \omega}(z)$ in $\operatorname{Sl}(2, \mathbb{C})$. The same pair produces a null curve $\Phi(z)=\mathbf{W}_{g, \omega}(z)$ in $\mathbb{C}^{3}$ by the Weierstraß formula (1.2). The two curves $\mathrm{F}(z)$ and $\Phi(z)$ are called cousins of each other. They share many properties. We mention only that the corresponding minimal surface in $\mathbb{R}^{3}$ and cmc-1-surface $\mathbb{H}^{3}$ are isometric. The construction of explicit expressions of cmc-1-cousins of a minimal surface is not possible in most cases. In other cases some efforts and elaborated techniques lead to interesting results. In [3] explicit but involved expressions for the cousins of the trinoid are obtained.

We show here an example, where the command S12CFrameBR finds the cmc-1-cousin of a minimal surface considered in [14] in connection with examples of cmc-1-surfaces with irregular ends. The Weierstraß data of this surface are

$$
g(z)=\frac{e^{2 z}(-1+z)}{1+z}, \quad \omega(z)=\frac{2(1+z)^{2}}{e^{2 z} z^{2}} .
$$

These are used as input data for the Bryant formula. Below are some Mathematica commands producing the explicit parametrization (7.3) of this curve curve and the final curve. The output of the command S12CFrameBR [g, omega] [z] is a huge expression that 

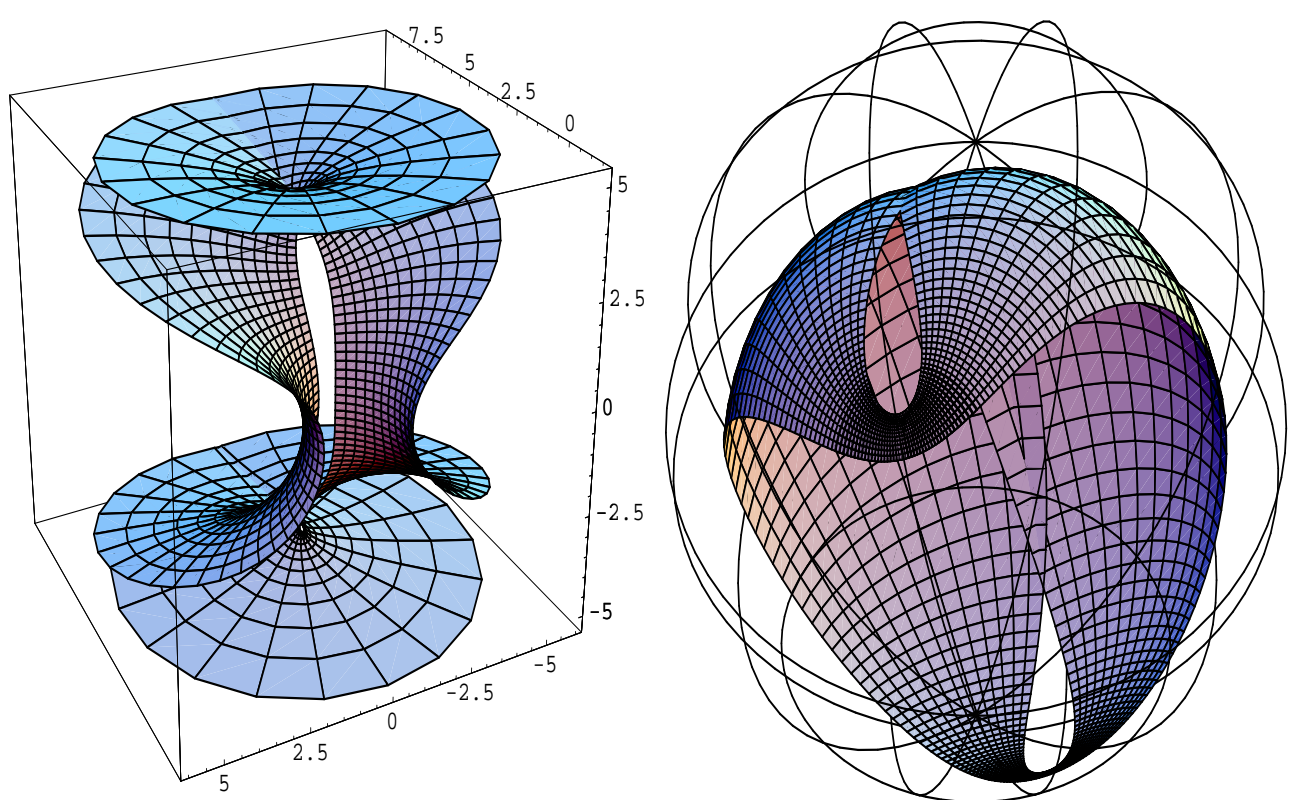

On the left the minimal surface in $\mathbb{R}^{3}$ corresponding to the Weierstraß data (7.2). Its cmc-1-cousin displayed in the upper half space model of $\mathbb{H}^{3}$ on the right.

is simplified with the commands PowerExpand and FullSimplify. The evaluation takes a few minutes. For a check we compute the Bryant data of the resulting expression.

\{omega $\left[z_{-}\right]=\left(2 *(1+z)^{\wedge} 2\right) / E^{\wedge}(2 * z) * z^{\wedge}-2, g\left[z_{-}\right]=E^{\wedge}(2 * z) *(z-1) /(1+z)$, $l c:=\left(\right.$ ZuhuanYuNullCurve0 $\left[z_{-}\right]=$Sl2CFrameBR $[g$, omega $][z]$;

ZuhuanYuNullCurve1 [z_] =ZuhuanYuNullCurve0 [Sqrt [5] *z]//Simplify;

ZuhuanYuNullCurve [z_]=ZuhuanYuNullCurve1 [z/Sqrt [5]]//FullSimplify)//Timing;

ZuhuanYuNullCurve [z]//PowerExpand//FullSimplify

\{bryantdata=nullcurveToBryantdata[ZuhuanYuNullCurve] [z]//FullSimplify,

$$
\mathrm{F}(z)=\frac{1}{5 \sqrt{2} z e^{z}}\left(\begin{array}{ccc}
\sqrt{5} \tau z+\sigma(4+5 z) & e^{2 z}(\sigma(4-5 z)+\sqrt{5} \tau z) \\
-5 \sigma z-\sqrt{5} \tau(4+5 z) & e^{2 z}(\sqrt{5} \tau(5 z-4)-5 \sigma z)
\end{array}\right)
$$

with $\sigma=\sinh (\sqrt{5} z), \tau=\cosh (\sqrt{5} z)$.

In general, it is easier to start with a null curve in $\mathrm{Sl}(2, \mathbb{C})$, determine its Bryant data and from these the corresponding null curve in $\mathbb{C}^{3}$. Next, we discuss an example based on the formula (4.3). In the special case $a(z)=e^{b(z)}$ the 1-form $\mu$ of (4.3) becomes $\mu=i\left(1+b^{2}\right) b^{-4} \mathrm{~d} b$ and Iwa $\mathbf{a}_{a, b}$ turns into the following purely algebraic formula:

$$
\mathbf{I w a}_{e^{b}, b}=\left(\begin{array}{cc}
b \cosh b & \frac{\cosh b+3 b(b \cosh b+\sinh b)}{3 i b^{2}} \\
i b \sinh b & \sinh b+\frac{3 b \cosh b+\sinh b}{3 b^{2}}
\end{array}\right) .
$$

The differential invariants of this curve can be computed with the methods of section 8.2. For the special choice $b(z) \rightarrow \sqrt{b(z)+2}$ we obtain the following simple expressions: Put 
$\widetilde{\mathbf{I w a}} \mathbf{a}_{b}=\mathbf{I w a}_{a, c}(z)$ with $a(z)=e^{\sqrt{b(z)+2}}$ and $c(z)=\sqrt{b(z)+2}$. The infinitesimal natural parameter and the null curvature of $\widetilde{\mathbf{I w a}} \mathbf{a}_{b}$ are

$$
\pi^{\prime}=\frac{\sqrt{b} \mathrm{~d} b}{2(b+2)} \quad \text { and } \quad \kappa(z)=-2-\frac{28}{b(z)^{3}}+\frac{4}{b(z)^{2}} .
$$

Note that for all choices of the function $b(z)$ these curves are reparametrizations of one of them, obtained for $b(z)=1$.

The following commands produce a 3-parameter family of null curves depending on 3 complex parameters $k, l, m$ and the output of the last gives the curve (7.4).

$\mathrm{a} 1\left[\mathrm{z}_{-}\right]=\mathrm{m} * \operatorname{Exp}[\mathrm{k} * \mathrm{a}[\mathrm{z}]] ; \mathrm{b} 1\left[\mathrm{z}_{-}\right]=1 * \mathrm{a}[\mathrm{z}]$;

$f f\left[a_{-}\right]\left[k_{-}, l_{-}, m_{-}\right]\left[z_{-}\right]=$Sl2CFrameIwasawa[a1, b1] $[z] / /$ FullSimplify;

ff $[a][1,1,1][z] / /$ FullSimplify;

We compute the Bryant data $g$ and $\omega$ of this curve. A suitable code for the Weierstraß formula (1.2) returns the minimal cousin:

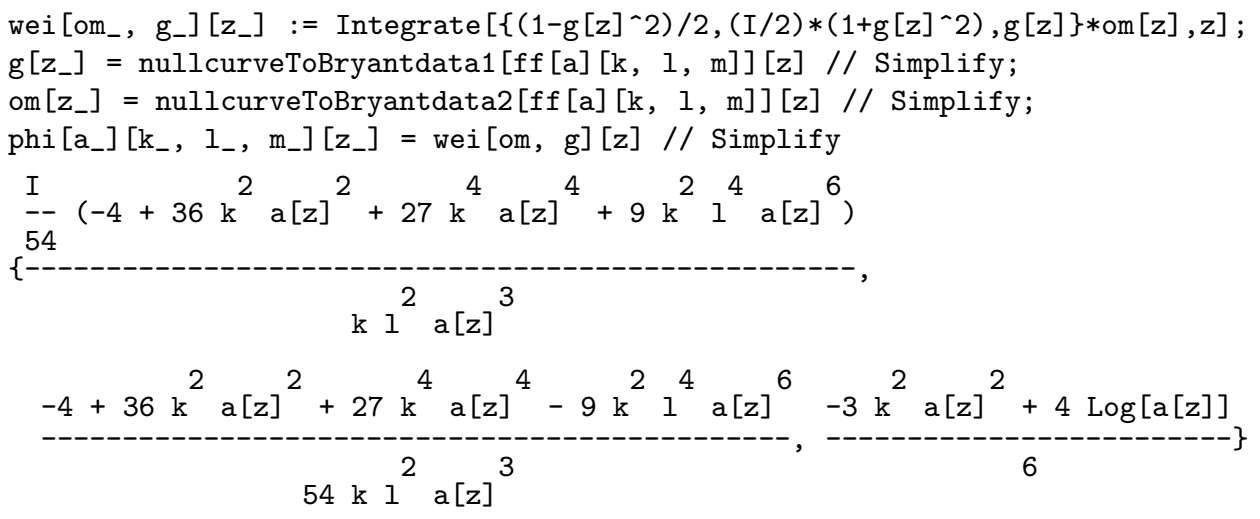

\section{Mathematica examples: plots and algebraic computations}

8.1. Two surfaces obtained with the Bianchi-Small formula BS. The Lorentzian model $\left\{\left(x_{0}, x_{1}, x_{2}, x_{3}\right) \in \mathbb{R}^{4} \mid-x_{0}^{2}+x_{1}^{2}+x_{2}^{2}+x_{3}^{2}=-1\right\}$ of $\mathbb{H}^{3}$ is bijectively mapped by $\left(x_{0}, x_{1}, x_{2}, x_{3}\right) \rightarrow\left(\begin{array}{cc}x_{0}+x_{3} & x_{1}+i x_{2} \\ x_{1}-i x_{2} & x_{0}-x_{3}\end{array}\right)$ to the set of all Hermitean matrices of determinant 1. A null curve $\mathrm{F}(z)$ is projected to a cmc-1-surface in this Hermitean model of $\mathbb{H}^{3}$ by $\mathbf{x}(u, v)=\mathrm{F}(u+i v) \mathrm{F}(u+i v)^{*}$, where $\mathrm{F}^{*}$ denotes the conjugate of the transposed matrix. The term directPlotBallmodelDisplay [ff] [u0,u1,v0,v1,pu,pv] of cmc1.m is a quick command generating plots of corresponding cmc-1-surfaces in the ball model of $\mathbb{H}^{3}$ for the parameter ranges $u_{0} \leq u \leq u_{1}, v_{0} \leq v \leq v_{1}$ with $p_{u}$ and $p_{v}$ being the number of plot points in $u$ - and $v$-direction respectively. This is done by computing the coordinates of the plot points of $\mathbf{x}(u, v)$ and transforming the numerical values directly to the ball model, avoiding in this way time consuming algebraic transformations of huge expressions. Several versions of this command, also related to the upper half space, are available. Options of Mathematica's ParametricPlot3D can be added at the end of the list of variables of directPlotBallmodelDisplay. 


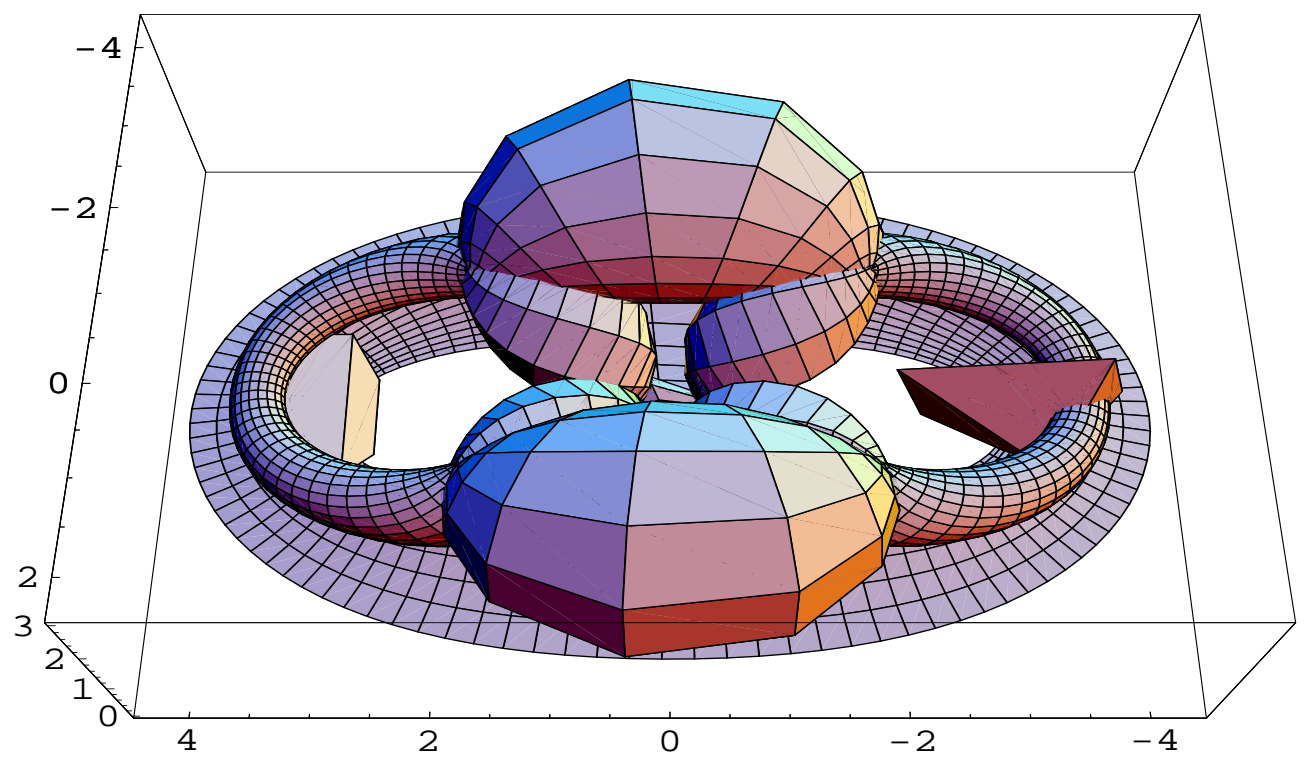

The cmc-1-surface corresponding to the functions (8.1) under BS in the upper half space model of $\mathbb{H}^{3}$

BianchiCaloSmallDouble $[\mathrm{f}, \mathrm{g}][\mathrm{z}]$ is our code for the Bianchi-Small formula (6.4). Let us consider the null curve corresponding under $\mathbf{B S}_{f, g}$ to the functions

$$
f(z)=\left(z^{4}\left(2 i+i z^{2}\right)^{2}\right)^{-1} \text { and } g(z)=z\left(z^{2}+i z+2\right)^{-1} .
$$

A plot of the corresponding cmc-1-surface in the upper half-space model is shown above. The surface shows interesting symmetries. The commands that generate this plot and the parametrization of the null curve are given below.

$\left\{\mathrm{g}\left[\mathrm{z}_{-}\right]=\mathrm{z} /\left(2+\mathrm{I} * \mathrm{z}+\mathrm{z}^{\wedge} 2\right), f\left[\mathrm{z}_{-}\right]=1 /\left(\mathrm{z}^{\wedge} 4 *\left(2 * I+I * \mathrm{z}^{\wedge} 2\right)^{\wedge} 2\right)\right\}$

ff $\left[z_{-}\right]=$BianchiCaloSmallDouble $[g, f][z] / / F u l l$ Simplify//PowerExpand//FullSimplify;

directPlotUpperhalfSpacePolar [ff] [1/3,4/3,-Pi, Pi , 33, 103, Axes->True,

ViewPoint $->\{0,1,2\}]$;

gwei $\left[z_{-}\right]=$nullcurveToBryantdata1 $[f f][z] / /$ FullSimplify

omwei $\left[z_{-}\right]=$nullcurveToBryantdata2 $[\mathrm{ff}][\mathrm{z}] / /$ FullSimplify

phi $\left[z_{-}\right]=$wei [gwei, omwei] [z] ;

ParametricPlot3D $[\mathrm{phi}[\mathrm{u}+\mathrm{I} * \mathrm{v}] / / \mathrm{Re} / /$ Evaluate $,\{\mathrm{u}, 0.3,0.45\},\{\mathrm{v}, \mathrm{r}=-1.6,-\mathrm{r}\}$, PlotPoints $->\{17,193\}$, PlotRange $->A l l$, ViewPoint $->\{3,0,-3.8\}]$;

Next, we choose the input data $f[z]$ and $g[z]$ as shown below, compute the null curve $\mathrm{ff}[z]$ and generate the plot. The differential invariants of this curve are computed. We show here the latter ones and the plot only.

$\left\{f\left[z_{-}\right]=\right.$Integrate $\left[\operatorname{Sin}[z]^{\wedge}-2, z\right], g\left[z_{-}\right]=$Integrate $\left.\left[\operatorname{Cos}[z]^{\wedge} 4, z\right]\right\}$;

ff $\left[z_{-}\right]=$BianchiCaloSmallDouble $[f, g][z] / /$ PowerExpand//FullSimplify;

directPlotBallmodelDisplay $[\mathrm{ff}][\mathrm{s}=-0.7,0.7,-3,3,43,73$, ViewPoint $->\{0,-1,-3\}]$;

nullcurveToBryantdata[ff] [z]

BianchiCaloSmallDoubleCanParameterPrime $[f, g][z]$

BianchiCaloSmallDoubleCurvature $[f, g][z] / /$ Simplify 


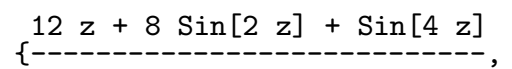

32

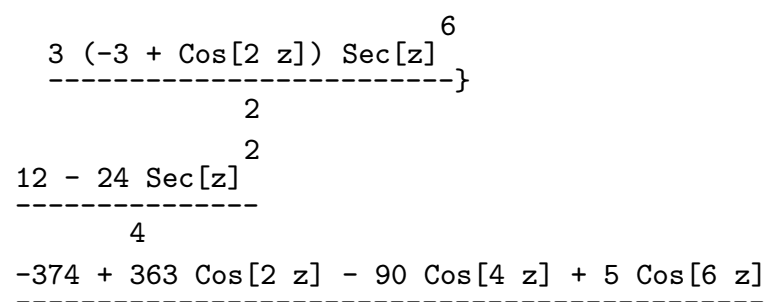

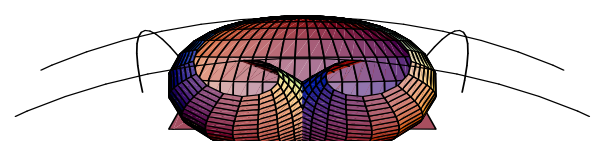

\section{z]}

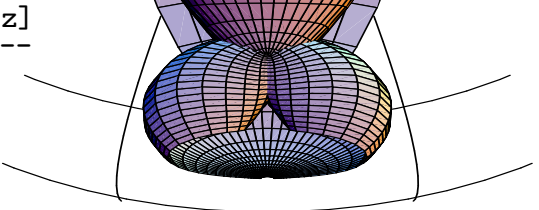

8.2. Bryant data, natural parameter and curvature. In this section we give two examples of curvature calculations. First we evaluate a Mathematica term defining a command Sl2FrameIwasawa [a,b] [z] for the integral representation formula (4.3). We suppose that Mathematica is already instructed with a command S12CIwasawaPar for the Iwasawa parametrization (2.3).

ff $\left[z_{-}\right]=S 12 C I$ wasawaPar $[a[z], b[z], c[z]]$;

$\mathrm{nn}\left[\mathrm{z}_{-}\right]=$Inverse $[\mathrm{ff}[z]] . f f^{\prime}[z] / /$ Simplify;

$\mathrm{gl}=\mathrm{nn}[\mathrm{z}] / /$ Det $/ /$ Simplify

op $\left[a_{-}, b_{-}\right]\left[z_{-}\right]=c^{\prime}[z] / /$.Flatten [Solve $\left.\left[g l==0, c^{\prime}[z]\right]\right]$

opint $\left[a_{-}, b_{-}\right]\left[z_{-}\right]:=$Integrate $[o p[a, b][z z], z z] / / . z z^{->z}$

S12FrameIwasawa $\left[a_{-}, b_{-}\right]\left[z_{-}\right]:=$

S12CIwasawaPar $[a[z], b[z]$, opint $[a, b][z]] / /$ Simplify

Next we compute the Bryant data, the squared infinitesimal natural parameter and the null curvature. The output of nullcurveCurvature [S12FrameIwasawa $[\mathrm{a}, \mathrm{b}]][\mathrm{z}] /$ is a huge expression that cannot be displayed here.

nullcurveToBryantdata[S12FrameIwasawa $[a, b]][z]$

nullcurveCanParameterPrime [S12FrameIwasawa [a,b]] [z]

nullcurveCurvature [S12FrameIwasawa $[a, b]][z] / /$ Timing;

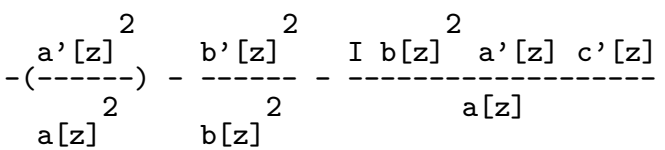

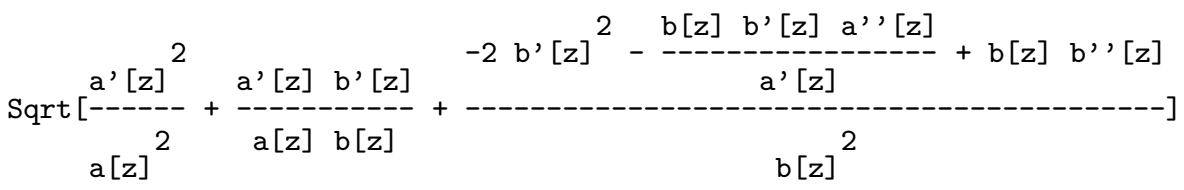

As a second example let us show the derivation of (6.8). We are using a utility toSchwarzian $[\mathrm{n}][\mathrm{f}, \mathrm{sf}][\mathrm{z}]$ of $\mathrm{cmc1.m}$ producing a rule that replaces the derivatives 
of order $3, \ldots, 3+n$ of a function $f(z)$ by corresponding derivatives of the expression $\left(3 f^{\prime \prime 2}(z)-(\mathcal{S}(f)(z))^{2} f^{\prime 2}(z)\right) / 2 f^{\prime}(z)$ obtained by solving the formula defining $\mathcal{S}(f)(z)$ for $f^{\prime \prime \prime}(z)$. The following evaluation lasts a few minutes and will finally return the expression (6.8).

BianchiCaloSmallDoubleCurv: $=($ Clear $[f, g, f f, b c s N C, b c s N C x]$;

ff $\left[z_{-}\right]=$BianchiCaloSmallDouble $[f, g][z]$;

deltaRule=Module $\left[\left\{\mathrm{rs}=\operatorname{Sqrt}[\mathrm{sg}[z]]^{\wedge} 2+4 \operatorname{delta}[z]\right]\right\}$,

Table $[D[s f[z],\{z, i\}]->$ Simplify $[D[r s,\{z, i\}]],\{i, 0,2\}]]$;

$\operatorname{bcsNC}\left[z_{-}\right]=$null curveCurvature $[f f][z]$;

SchwarzianRule $=\{$ toSchwarzian [3] [f,sf] [z], toSchwarzian [3] [g,sg] [z] $\} / /$ Flatten;

bcsNCx= (bcsNC//. SchwarzianRule) $/ /$ Simplify;

(bcsNCx//.deltaRule)//Simplify//Expand)

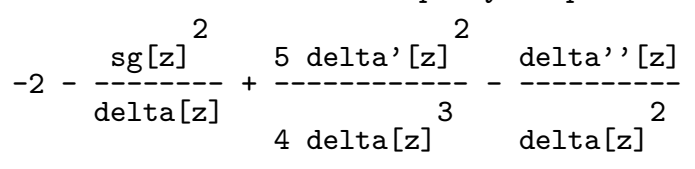

The command BianchiCaloSmallDoubleCurvatureFORMAL[sf,sg] [z] encodes the formula (6.8) in formal terms $\mathrm{sf}[\mathrm{z}], \mathrm{sg}[\mathrm{z}]$ that stand for the Schwarzians and schwarzderbr $[f][z]$ encodes the Schwarzian. An evidence of the parameter invariance of (6.8) is obtained with

BianchiCaloSmallDoubleCurvatureFORMAL [

schwarzderbr $[f[t[\#]] \&]$, schwarzderbr $[g[t[\#]] \&]][z]-$

BianchiCaloSmallDoubleCurvatureFORMAL [

schwarzderbr $[f]$, schwarzderbr $[g]][t[z]] / /$ Simplify

8.3. A cmc-1-surface established with the algebraic formula $\mathbf{M}_{f, g}$. The following surface is produced by the formula $\mathbf{M}_{a, b}^{(n, k, l)}$ (see (5.5)) for $n=3, k=l=1,\left(a(z)=e^{z}, b(z)=\right.$ $e^{z} / z$ (Mathematica code S12CFrameMeromorphic):

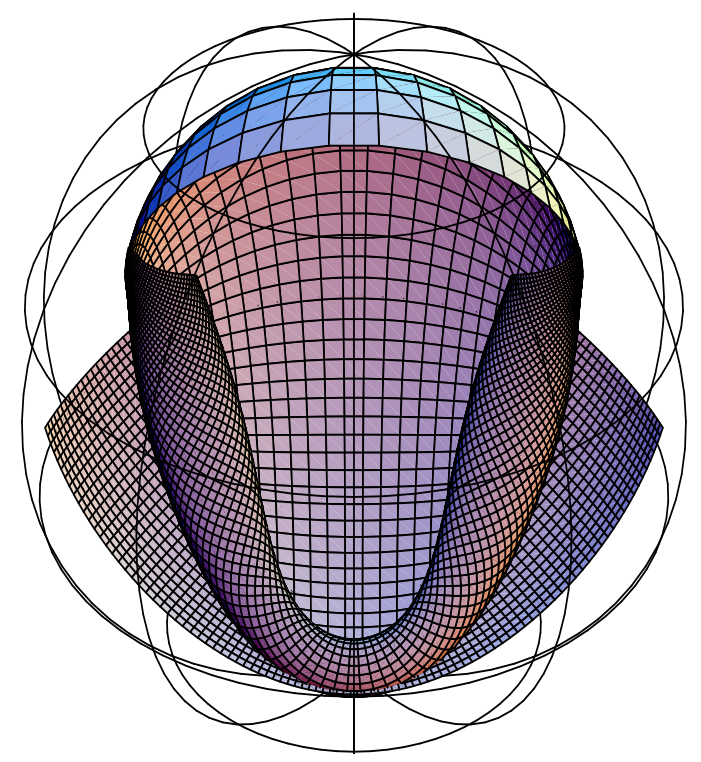


ff $\left[z_{-}\right]=$S12CFrameMeromorphic $[\operatorname{Exp}[\#] \&, \operatorname{Exp}[\#] / \# \&][z]$

directPlotBallmodelDisplay $[f f][-1,1.5,-2,2,67,97$, ViewPoint-> $\{3,0,2\}]$;

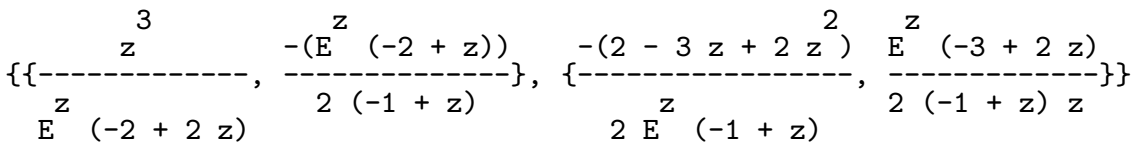

\section{References}

[1] R. Aiyama and K. Akutagawa, Kenmotsu type representation formula for surfaces with prescribed mean curvature in the 3-sphere, Tohoku Math. J. 52 (2000), 95-105.

[2] L. Bianchi, Lezioni di Geometria Differenziale, 3rd ed., Nicola Zanichelli, Bologna, 1927.

[3] A. I. Bobenko, T. V. Pavlyukevich and B. A. Springborn, Hyperbolic constant mean curvature one surfaces: Spinor representation and trinoids in hypergeometric functions, math.DG/0206021.

[4] R. Bryant, Surfaces of mean curvature one in hyperbolic space, Sémin. Palaiseau/France 1983/1984, Astérisque 154-155 (1988), 321-347.

[5] H. Gollek, Deformations of minimal curves in $\mathbb{C}^{3}$, in: Proc. 1-st NOSONGE Conference, Warsaw, Sept. 1996.

[6] H. Gollek, Deformations of minimal Surfaces containing planar Geodesics, in: Proc. 18-th Winter School "Geometry and Physics" (Srni, 1998).

[7] H. Gollek, Natural algebraic representation formulas for curves in $\mathbb{C}^{3}$, Banach Cent. Publ. 57 (2002), 109-134.

[8] M. Kokobu, M. Takahashi, M. Umehara and K. Yamada, An analogue of minimal surface theory in $\operatorname{Sl}(n, \mathbb{C}) / \mathrm{SU}(n)$, Trans. Am. Math. Soc. 354 (2001), 1299-1325.

[9] M. Kokubu, M. Umehara and K. Yamada, An elementary proof of Small's formula for null curves in PSL(2, C) and an analogue for Legendrian curves in $P S L(2, \mathbb{C})$, Osaka J. Math. 49 (2003), 697-715.

[10] L. L. de Lima and P. Roitman, Constant mean curvature one surfaces in hyperbolic 3space using the Bianchi-Calò method, Anais de Academia Brasileira de Ciências 74 (2002), $19-24$.

[11] A. J. Small, Surfaces of constant mean curvature 1 in $\mathbb{H}^{3}$ and algebraic curves on a quadric, Proc. AMS 122 (1994), 1211-1220.

[12] W. Walter, Gewöhnliche Differentialgleichungen, Springer, 1993.

[13] M. Umehara and K. Yamada, Another construction of a CMC-1 surface in $\mathbb{H}^{3}$, Kyungpook Math. J. 35 (1996), 831-849.

[14] Z. H. Yu, Surfaces of constant mean curvature one in the hyperbolic three-space with irregular ends, Tohoku Math. J. 53 (2001), 305-318. 